\title{
Behavioural and neural indices of perceptual decision-making in autistic children during visual motion tasks
}

Catherine Manning a, ${ }^{\text {* }}$, Cameron D. Hassall ${ }^{\mathrm{c}}$, Laurence T. Hunt ${ }^{\mathrm{c}}$, Anthony M. Norcia ${ }^{\mathrm{d}}$, Eric-Jan Wagenmakers ${ }^{\mathrm{e}}$, Nathan J. Evans ${ }^{\mathrm{f} \dagger}$ \& Gaia Scerif ${ }^{\mathrm{a} \dagger}$

${ }^{a}$ Department of Experimental Psychology, University of Oxford, UK

${ }^{\mathrm{b}}$ School of Psychology and Clinical Language Sciences, University of Reading, UK

${ }^{c}$ Department of Psychiatry, University of Oxford, UK

${ }^{\mathrm{d}}$ Department of Psychology, Stanford University, USA

${ }^{\mathrm{e}}$ Faculty of Social and Behavioural Sciences, University of Amsterdam, The Netherlands

${ }^{\mathrm{f}}$ School of Psychology, University of Queensland, Australia

* This manuscript has not undergone peer review *

* Corresponding author: Catherine Manning; c.a.manning@ reading.ac.uk

$\dagger$ Nathan J. Evans and Gaia Scerif contributed equally to this work. 


\begin{abstract}
.
Many studies report atypical responses to sensory information in autistic individuals, yet it is not clear which stages of processing are affected, with little consideration given to decisionmaking processes. We combined diffusion modelling with high-density EEG to identify which processing stages differ between 50 autistic and 50 typically developing children aged 6-14 years during two visual motion tasks. Our pre-registered hypotheses were that autistic children would show task-dependent differences in sensory evidence accumulation, alongside a more cautious decision-making style and longer non-decision time across tasks. We tested these hypotheses using hierarchical Bayesian diffusion models with a rigorous blind modelling approach, finding no conclusive evidence for our hypotheses. Using a data-driven method, we identified a response-locked centro-parietal component previously linked to the decision-making process. The build-up in this component did not consistently relate to evidence accumulation in autistic children. This suggests that the relationship between the EEG measure and diffusion-modelling is not straightforward in autistic children. Compared to a related study of children with dyslexia, motion processing differences appear less pronounced in autistic children. Our results also provide weak evidence that ADHD symptoms moderate perceptual decision-making in autistic children.
\end{abstract}




\section{Introduction}

Autism is a developmental condition affecting social communication and interaction as well as non-social domains, such as sensory functioning ${ }^{1}$. Atypical responses to sensory information are well-documented in autism, ranging from first-hand reports of sensory processing differences ${ }^{\text {e.g., }}{ }^{2-4}$ to evidence of altered behavioural and physiological responses to sensory stimuli (see [5], for review). Empirical investigations of sensory processing often use psychophysical thresholds to quantify differences between autistic and non-autistic individuals. Here, researchers estimate the stimulus level (or difference between stimuli) required to give rise to a given level of accuracy for each individual, and compare these estimates between groups. Reports of group differences in psychophysical thresholds range from reduced sensitivity ${ }^{\text {e.g., }}{ }^{6-8}$ to enhanced sensitivity e.g., $9-11$, depending on the task. One key unresolved issue is the level of processing at which group differences in psychophysical thresholds arise. It is often assumed that differences in psychophysical thresholds reflect differences in sensitivity, but they could also reflect differences in decision-making strategies (for example, requiring more or less information before making a response). Moreover, research using psychophysical thresholds potentially neglects group differences in speedaccuracy tradeoffs, by failing to account for differences in response time.

Diffusion models allow both accuracy and response time to be analysed, in order to decompose performance into distinct processing stages ${ }^{12,13}$ (see [14], for review and Figure 1 for a graphical representation of multiple parameters feeding into decisions). The decision is modelled as a noisy evidence accumulation process from a starting point towards one of two decision bounds (e.g., "left" or "right" decision bounds for a motion discrimination task). The main parameters of the model are drift-rate, boundary separation, starting point and nondecision time. The drift-rate reflects the rate of evidence accumulation, with information being accumulated more rapidly in more sensitive individuals and in conditions with stronger 
sensory evidence. Boundary separation reflects how far apart the decision boundaries are, and thus how much accumulated evidence is required before making a decision. The boundary separation parameter reflects speed-accuracy tradeoffs, with individuals with narrow decision bounds making more 'risky' decisions, prioritising speed over accuracy, and individuals with wider decision bounds making more cautious decisions. The starting point can reflect bias, if the evidence accumulation process starts nearer to one of the bounds than the other. The nondecision time reflects sensory encoding and response generation that happen outside of the decision process, but contribute to the overall response time. Modelling accuracy and response time data together may be particularly useful for understanding conditions such as autism, as the resulting parameters may be more sensitive to group differences than accuracy or response time alone ${ }^{15,16}$. Importantly, it is possible to investigate group differences in sensitivity (drift rate) while controlling for potential differences in speed-accuracy tradeoffs.

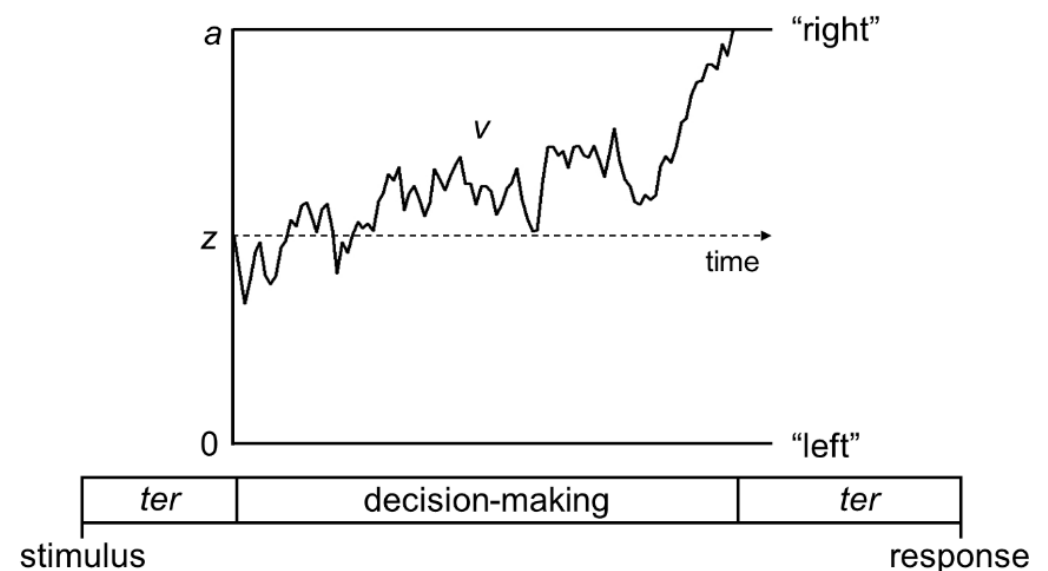

\section{Figure 1. Schematic representation of the main diffusion model parameters}

Within the diffusion model, the decision-making process is represented as a noisy evidence accumulation process originating from a starting point, $z$, towards one of two decision bounds (e.g., "left" and "right" directions in the motion tasks used in this study). Boundary separation, $a$, reflects the distance between the two bounds, indexing response caution. Drift-rate, $v$, is the rate of evidence accumulation. Non-decision time, ter, is the time taken for non-decision processes including sensory encoding and response generation. Figure reproduced from https://osf.io/rqw62/ under a CC-BY4.0 License.

The utility of this approach for understanding autistic perception has recently been shown in a study of orientation discrimination by Pirrone et al. ${ }^{17}$. In this study, autistic adults 
had longer response times, but similar accuracies, compared to non-autistic individuals. Diffusion modelling revealed that autistic participants responded more cautiously (i.e., had wider decision bounds) and had longer non-decision times than non-autistic individuals, yet the groups did not differ in terms of drift rate, suggesting similar levels of sensitivity to orientation information. Without such modelling, it may have been concluded from autistic individual's slower responses that they were less sensitive to orientation information. The finding of increased response caution was later replicated in a study of orientation discrimination in autistic children aged 6 to 16 years ${ }^{18}$, although in this study, there was no evidence for differences between autistic and typically developing children in non-decision time, and it was not possible to investigate differences in drift-rates between the groups as difficulty levels were varied for each participant.

Three further studies have used the diffusion model with autistic populations to understand stereotypical attitudes in an implicit association task ${ }^{19}$, performance in the correct 'go' trials of a response inhibition stop-task ${ }^{20}$ and numerical addition problems ${ }^{21}$. In all studies, autistic individuals were reported to display a more cautious response style than nonautistic individuals, in line with the findings of Pirrone et al. ${ }^{17,18}$. Conversely, none of the studies found evidence of differences in non-decision times between autistic and non-autistic individuals. Karalunas et al. ${ }^{20}$ reported that autistic individuals had reduced drift rates compared to non-autistic individuals ${ }^{\text {c.f. }}{ }^{17}$, but Kirchner et al. ${ }^{19}$ and Iuculano et al. ${ }^{21}$ found no group differences in this parameter. Combining the results of these studies together, it seems that differences in boundary separation between autistic and non-autistic individuals may be ubiquitous across tasks, while differences in drift-rate and non-decision time might depend on the task and age of participants.

Given the range of differences reported in psychophysical thresholds in autistic individuals $^{\text {e.g., }}{ }^{-11}$, the diffusion model approach should be extended to understand autistic 
performance across diverse perceptual tasks. In this study, we applied the diffusion model to offer new insights into a well-studied aspect of autistic perception: visual motion processing. We presented two visual motion tasks that have been linked to either increased ${ }^{22}$ or reduced $^{23,24}$ sensitivity in autism in studies assessing psychophysical thresholds. The reason for choosing these tasks was that we expected different effects on diffusion model parameters between groups in these tasks, making them particularly informative for understanding the generality of differences in perceptual decision-making in autism. Additionally, we combined the diffusion model approach with electroencephalographic (EEG) data because both techniques investigate multiple processing stages and temporal dynamics that are not captured by simple accuracy indices and therefore have the potential to provide novel, multilevel insights into motion processing in autism (see [25]).

The first task was a motion coherence task, which has been used extensively with autistic populations (see [26], for review). In this task, participants are required to discriminate the direction of motion carried by coherently moving signal dots in a field of randomly moving noise dots ${ }^{22-24}$. The second task was a direction integration task in which participants are required to discriminate the average direction of dots moving in directions sampled from a Gaussian distribution, with difficulty being manipulated by increasing the variability of dot directions ${ }^{22,27}$. These tasks were previously presented to autistic and nonautistic children with surprising results ${ }^{22,27}$. Instead of showing difficulties in both tasks, as expected from accounts of impaired dorsal-stream functioning ${ }^{28}$ or difficulties integrating information $^{29}$, the autistic children demonstrated enhanced performance in the direction integration task compared to typically developing children. In the motion coherence task, the autistic and non-autistic children performed similarly. This pattern of results suggested that while autistic children demonstrated increased integration of motion information compared to typically developing children, they may be limited in the motion coherence task due to 
difficulties filtering out the randomly moving noise dots ('noise exclusion'; see also [30,31]). In this study we applied diffusion models to performance in these two tasks to understand more about the underlying processes that are atypical in autistic individuals.

We pre-registered four hypotheses prior to completing data collection and prior to data analysis (https://osf.io/znyw2):

1. Autistic children will have reduced drift-rates in the motion coherence task compared to typically developing children, in line with previous reports of reduced motion coherence sensitivity in autistic individuals e.g., 23,24,32.

2. Autistic children will have increased drift-rates in the direction integration task compared to typically developing children, based on previous reports of enhanced discrimination performance in this task ${ }^{22,27}$.

3. Autistic children will show wider response boundaries compared to typically developing children in both motion coherence and direction integration tasks ${ }^{17-21}$.

4. Autistic children will show longer non-decision times than typically developing children in both motion coherence and direction integration tasks, following Pirrone et al. ${ }^{17}$. Other studies have reported no differences in non-decision time between autistic and typical individuals ${ }^{19,20}$, but we reasoned that the visual discrimination task used by Pirrone et al. was more similar to our tasks. However, we note that a study comparing autistic and typically developing children on an orientation discrimination task was published since pre-registering our hypotheses showing no group differences in non-decision times ${ }^{18}$.

These hypotheses were addressed by models fit to the behavioural data. High-density EEG data were collected from the majority of participants, in order to conduct exploratory investigations into links between the model parameters and EEG markers. Owing to its high temporal resolution, EEG provides potentially complementary insights into the stages of 
processing that might be affected in autism, allowing links to be made between brain and behaviour. For example, a response-locked neural marker of the decision-making process that correlates with drift rate has been identified in both typically developing children ${ }^{33}$ and children with dyslexia ${ }^{34}$, with children with dyslexia showing a reduced drift-rate compared to typically developing children, and corresponding reductions in the neural marker. An analysis of evoked potentials locked to the global motion onset in a subset of autistic participants revealed no differences in early neural responses to motion onset, but suggested that later processing stages may be affected ${ }^{35}$. We therefore focus on response-locked markers of the decision-making process in this study.

\section{Methods}

Blind modelling and sampling plan

Due to difficulties in specifying all modelling decisions before seeing the data, we used a blind modelling approach ${ }^{36}$, with an author who was not involved in data collection (N.J.E.) acting as a blind modeller (see pre-registration document: https://osf.io/znyw2). The blind modeller made all modelling decisions (e.g., removal of outliers, specification of priors) in blinded datasets prepared by the first author (C.M.) in which the key variable relevant to the hypotheses under test (group membership) was randomly permuted.

Monte Carlo simulations ${ }^{37}$ suggested that 49 participants per group were required on average to detect a moderate effect size of $d=0.5$. We followed a 2-stage analysis plan to aim for confidence in our results while minimising unnecessary testing of participants. In the first stage, we analysed the data from groups of 40 autistic and 40 typically developing children. The first 40 participants in each group were not well-matched in performance IQ, so we tested another 10 typically developing children. We then selected the 40 typically 
developing children who best matched the 40 autistic children in age and performance IQ, using the R MatchIt package ${ }^{38}$. The blind modeller analysed the blinded dataset (analysis code here: https://osf.io/f3wnt/ ) and the first author then ran the analysis on the unblinded dataset. In the absence of strong evidence for our hypotheses (Bayes factors (BF) between 1/6 and 6), we proceeded to the second stage of our analysis plan. We resumed data collection until we had groups of 60 typically developing children and 50 autistic children. We selected the 50 typically developing children who best-matched the 50 autistic children in terms of age and performance IQ. The blind modeller again analysed the blinded dataset (analysis code here: https://osf.io/f3wnt/), before the final unblinded analyses were performed by the first author.

\section{Participants}

Following the steps outlined in our sampling plan, our final dataset included 50 autistic children (40 males) and 50 typically developing children (27 males). All children included in the dataset had normal or corrected-to-normal acuity, as measured by a Snellen acuity chart, and no evidence of intellectual disability (verbal IQ > 70 and performance IQ > 70 on the Wechsler Abbreviated Scales of Intelligence, $2^{\text {nd }}$ edition [WASI-II $\left.{ }^{39}\right]$ ). All typically developing children included in the dataset scored below the cut-off for autism $(<15)$ on the Social Communication Questionnaire $\left(\mathrm{SCQ}^{40}\right)$ completed by parents. All autistic children included in the dataset had an independent clinical diagnosis of autism, and scored above the cut-off for an autism spectrum condition on the Social Communication Questionnaire $(\geq 15)$ and/or on the Autism Diagnostic Observation Schedule, $2^{\text {nd }}$ edition (ADOS-2 ${ }^{41}$ ) (see $\left.[22,27]\right)$. Demographics for the participants included in the dataset are presented in Table 1. EEG data were collected during task performance in a subset of these children: in the motion coherence task we had EEG data for 46 typically developing and 43 autistic children and in the direction 
integration task we had EEG data for 45 typically developing and 45 autistic children. The EEG data from some of these participants has been included in a paper investigating responses locked to the onset of global motion in autistic and dyslexic children ${ }^{35}$. Datasets from an additional 2 typically developing children were excluded due to poor visual acuity ( $n$ $=1)$ or not meeting the criterion of correct responses in the practice phase $(n=1)$, and datasets from an additional 7 autistic children were excluded due to poor visual acuity $(n=$ 1), having an IQ below $70(n=2)$ or not meeting the autism cut-offs in either the SCQ or ADOS $(n=4)$. Many of the typically developing children overlapped with those in the control group in a related study of dyslexia ${ }^{34}$. As we expected a relationship between dyslexia and perceptual decision-making ${ }^{42}$, we measured reading ability with the Test of Word Reading Efficiency $-2^{\text {nd }}$ Edition (TOWRE- $2^{43}$ ) and measured spelling ability using the Wechsler Individual Achievement Test (WIAT-III ${ }^{44}$ ) in all participants (see Table 1 for standard scores). As decision-making parameters have also been shown to diverge in children with inattentive and hyperactive symptoms ${ }^{20,45}$, we also collected parent-report ratings on 9 items assessing children's inattention and 9 items assessing hyperactivity/impulsivity, from the DSM-IV ${ }^{46}$ criteria for ADHD as used in the Swanson, Nolan and Pelham-IV Questionnaire (SNAP-IV ${ }^{47}$;

https://www.amerihealth.com/pdfs/providers/resources/worksheets/prevhealth_swan.pdf). These items were binary coded and an average item score was extracted for each of the inattention and hyperactivity/impulsivity dimensions. As shown in Table 1, the autistic children had higher scores than typically developing children on both dimensions.

Table 1. Demographics of participants included in final dataset

$$
\begin{array}{ll}
\text { Typically developing } & \text { Autistic } \\
(\mathrm{n}=50) & (\mathrm{n}=50)
\end{array}
$$




\begin{tabular}{lll}
\hline Age & $10.61(2.34) 6.55-14.98$ & $10.91(2.43) 6.54-14.94$ \\
Performance IQ & $110.44(13.00) 81-145$ & $104.98(14.99) 78-138$ \\
Verbal IQ & $110.90(9.64) 95-142$ & $104.28(14.86) 76-137$ \\
Full-scale IQ & $112.06(10.49) 89-142$ & $105.22(15.09) 76-133$ \\
SCQ & $2.46(2.44) 0-11$ & $20.45(7.12) 6-36$ \\
ADOS-2 - Total & $110.42(16.27) 81-146$ & $94.70(22.81) 50-152$ \\
WIAT III Spelling & $105.02(10.17) 80-135$ & $96.96(18.69) 55-132$ \\
TOWRE-2: Phonemic & & \\
Decoding Efficiency & $0.17(0.23) 0-1$ & $0.58(0.29) 0-1$ \\
Inattentiveness & $0.08(0.19) 0-1$ & $0.55(0.34) 0-1$ \\
Hyperactivity/impulsivity 0 &
\end{tabular}

Note. Data are presented as M (SD) Range. SCQ = Social Communication Questionnaire ${ }^{40}$. ADOS-2 = Autism Diagnostic Observation Schedule, $2^{\text {nd }}$ Edition ${ }^{41}$. WIAT-III $=$ Wechsler Individual Achievement Test, 3rd Edition ${ }^{44}$. TOWRE-2 = Test of Word Reading Efficiency, $2^{\text {nd }}$ edition ${ }^{43}$. Standard scores are presented for the WIAT-III and TOWRE-2. Average item scores are presented for the Inattentiveness and Hyperactivity/impulsivity scales.

\section{Apparatus}

Stimuli were presented on a Dell Precision M3800 laptop (2048 x 1152 pixels, $60 \mathrm{~Hz}$ ) using the Psychophysics Toolbox for MATLAB ${ }^{48,49}$. EEG signals were collected with 128 channel Hydrocel Geodesic Sensor Nets connected to Net Amps 300 (Electrical Geodesics Inc., OR, USA) and NetStation 4.5 software. A photodiode attached to the monitor independently verified the timing of stimulus presentation. Participants provided responses on a Cedrus RB-540 response box (Cedrus, CA, USA). 


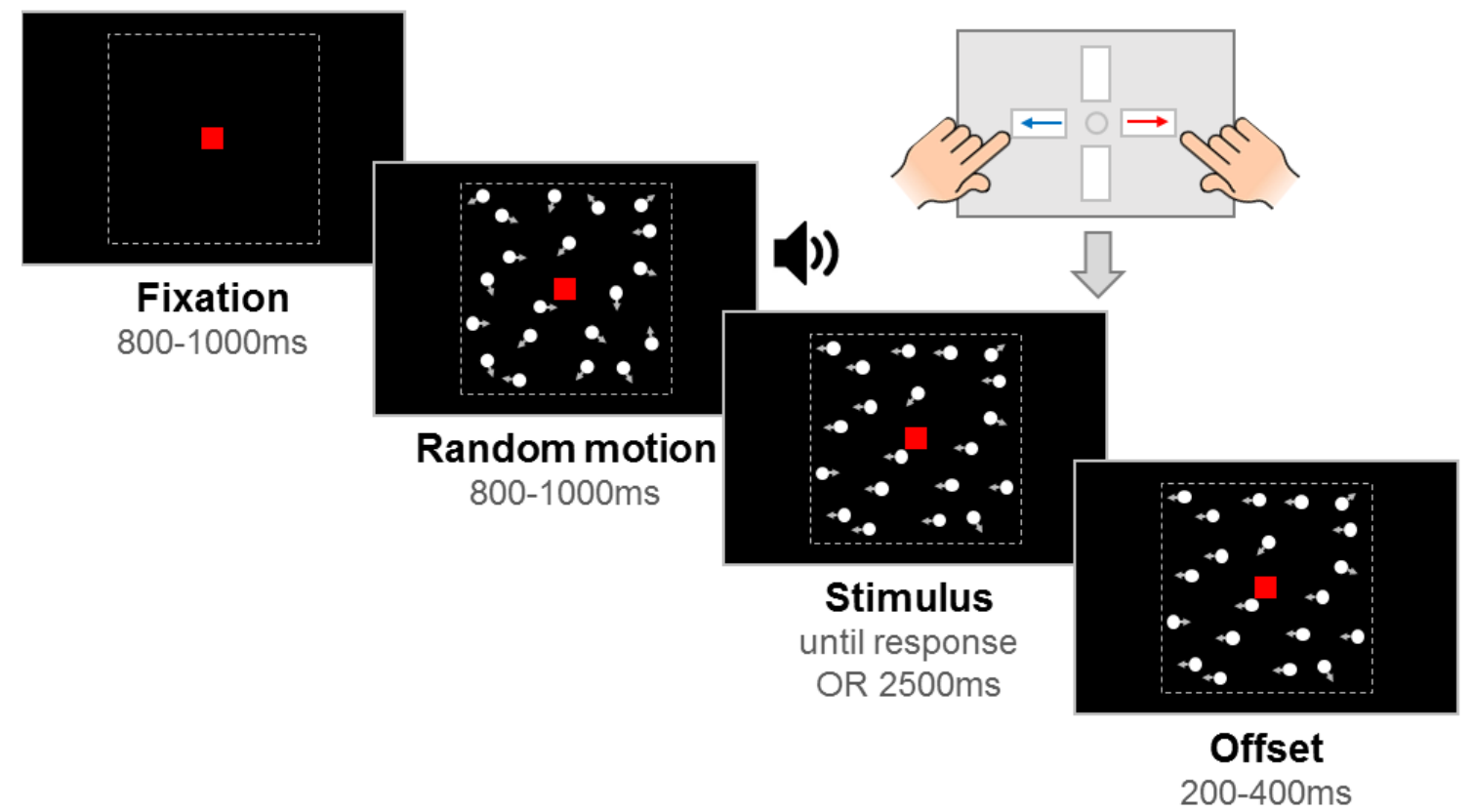

Figure 2. Schematic representation of trial procedure.

An initial fixation period was followed by a random motion period consisting of randomly moving dots. Next, the stimulus period contained leftward or rightward global motion and lasted until the child reported the direction with a response box. If there was no response, the stimulus remained on the screen for $2500 \mathrm{~ms}$. The stimulus remained on the screen for an offset period after the response or after the maximum stimulus duration was reached. Note that arrows (indicating movement) and dotted lines (marking the square stimulus region) are shown for illustrative purposes only. Figure reproduced from https://osf.io/wmtpx/ under a CC-BY4.0 license.

\section{Stimuli}

Stimuli comprised of 100 white, randomly positioned dots (diameter $0.19^{\circ}$ ) moving inside a square aperture $\left(10^{\circ} \times 10^{\circ}\right)$ at $6^{\circ}$ s on a black screen. The dots had a limited lifetime of $400 \mathrm{~ms}$. A red fixation square $\left(0.24^{\circ} \times 0.24^{\circ}\right)$ was presented throughout the trial. Each trial consisted of a fixation period, a random motion period, a stimulus period, and an offset period (see Figure 2). The fixation period had a randomly selected duration between 800 and $1000 \mathrm{~ms}$. The random motion period presented the stimulus dots moving in random, incoherent directions, for a randomly selected duration between 800 and $1000 \mathrm{~ms}$. The random motion period ensured that pattern- and motion-onset evoked potentials were distinguished temporally from the onset of directional motion in the stimulus period. At the start of the stimulus period, we presented an auditory tone to signal to participants that the 
directional motion had started. In the motion coherence task, directional motion (leftward or rightward) was introduced in a proportion of 'signal' dots, while the remainder of the dots continued to move in random directions. In the direction integration task, the directions of dots in the stimulus phase were distributed according to a Gaussian distribution with a mean leftward or rightward direction. The stimulus period was presented until a response was made, or until $2500 \mathrm{~ms}$ had elapsed. Finally, the offset period continued the directional motion for a randomly selected duration between $200-400 \mathrm{~ms}$, which served to dissociate response-locked EEG activity from that associated with motion offset.

\section{Experimental task procedure}

Children were presented with both motion coherence and direction integration tasks the order of which was counterbalanced between participants. The tasks were presented in the context of a child-friendly game set in 'Insectland' (based on [33]). Using animations, it was explained to participants that the fireflies were escaping from their viewing boxes, and that the zookeeper needed to know through which side of the box the fireflies were escaping, so that he could fix the box and 'save' the fireflies. Participants were told that there were 10 different viewing boxes, corresponding to 10 different 'levels' of the game. Levels 1-5 corresponded to one task (either motion coherence or direction integration), and Levels 6-10 corresponded to the other task, depending on the counterbalancing order for each participant. Levels 1 and 6 were practice phases, and the experimental blocks were presented in the remaining 4 levels for each task. In the motion coherence task, difficulty was manipulated by varying the proportion of dots moving coherently, and in the direction integration task, difficulty was manipulated by varying the standard deviation of the distribution from which the dot directions were sampled. 
The practice phases began with four demonstration trials with no random motion phase and an unlimited stimulus phase, to introduce the task to participants. Participants used the 'left' and 'right' arrow keys on the response box to report the direction. The first two demonstration trials were designed to be 'easy' (100\% coherence or $1^{\circ}$ standard deviation), while the last two were more difficult $\left(75 \%\right.$ and $50 \%$ coherence, or $10^{\circ}$ and $25^{\circ}$ standard deviations). Next, there were up to 20 criterion trials (coherence of $95 \%$ or $5^{\circ}$ standard deviation), which included the boil phase. It was explained to participants that the fireflies would be going "all over the place" at first, so that they must wait for the fireflies to escape before responding. They were told that there would be an alarm (auditory beep) to help them, and that they must wait for this alarm before deciding which way the fireflies were escaping. This auditory cue removed temporal uncertainty about the onset of the stimulus phase. There was a time limit of $2500 \mathrm{~ms}$, with visual feedback presented if participants did not respond within this time ("Timeout! Try to be quicker next time!", presented in red text). When participants met a criterion of four consecutive correct responses, they moved on to eight practice trials which increased in difficulty (motion coherence task: $80 \%, 70 \%, 60 \%, 50 \%$, $40 \%, 30 \%, 20 \%, 10 \%$; direction integration task: $5^{\circ}, 10^{\circ}, 15^{\circ}, 20^{\circ}, 30^{\circ}, 40^{\circ}, 50^{\circ}, 60^{\circ}$ ). Participants were reassured that it was fine if they got some of these wrong and/or if they had to guess. Visual feedback was presented after each trial throughout Level 1 ("That was correct!" presented in green text, or "It was the other way that time" or "Timeout! Try to be quicker next time!", presented in red text). For 1 child, Level 1 was repeated as they did not meet the criterion of four consecutive correct responses on the first attempt but did on the second.

Levels 2-5 and 7-10 each consisted of 38 trials (totalling 152 trials for each task), with 9 repetitions for each of two difficulty levels (motion coherence task: 30\%, 75\%; direction integration task: $70^{\circ}, 30^{\circ} \mathrm{SD}$ ) and each motion direction (leftward, rightward), and 2 catch 
trials presenting $100 \%$ coherent $\left(0^{\circ} \mathrm{SD}\right)$ motion. The difficulty levels were chosen through piloting, to ensure that children of all ages would be able to complete the task, while still obtaining enough errors for diffusion modelling. No trial-by-trial feedback was presented in the experimental phase, apart from a 'timeout' message (as before) if no response was given within $2500 \mathrm{~ms}$ after the stimulus period began. At the end of each level, participants were presented with their 'points' for the preceding block of trials. Participants were told that they would get points for both speed and accuracy. The points reflected an efficiency score, computed by $(1 /$ median $\mathrm{RT}) *$ number of correct responses $* 2$, rounded to the nearest integer. In the event that participants obtained a score under 10, a score of 10 points was given to avoid demotivation. Trials were presented automatically, but the experimenter had controls to pause and resume trial presentation (see below). See https://osf.io/f3wnt/ for experimental code.

\section{General procedure}

Ethical approval was provided by the Central University Research Ethics Committee at the University of Oxford (R56348/RE004) and the study was conducted in accordance with this approval. Parents/carers of child participants provided written informed consent, and children provided verbal or written assent. Most children took part at the University of Oxford, although one child took part at home without EEG. Participants initially completed a Snellen acuity test to confirm normal or corrected-to-normal vision. During the experimental tasks, participants sat $80 \mathrm{~cm}$ away from the computer screen in a dimly lit room. For children who were willing to take part with EEG, we fitted the net prior to the experiment and ensured that electrode impedances were below $50 \mathrm{k} \Omega$. EEG data were acquired at a sampling rate of $500 \mathrm{~Hz}$ with a vertex reference. 
Children were monitored by an experimenter during the tasks. The experimenter gave regular encouragement and task reminders, pausing before starting a trial where necessary (e.g., to remind the child to stay still). Children had short breaks at the end of each block (or 'level'), and a longer break after completing the first task (i.e., at the end of 'level 5'). The electrode impedances were re-assessed for children wearing EEG nets during this longer break. Children recorded their progress through the levels using a stamper and a record card. The children also completed the WASI-II, TOWRE-2 and the WIAT-III spelling subtest. The autistic children also completed module 3 or 4 of the ADOS-2. The whole session took no longer than 2 hours for typically developing children and no longer than 3 hours for autistic children. Children were given a $£ 10$ voucher to thank them for their time.

\section{Drift-diffusion modelling of data}

Trials with RTs under $200 \mathrm{~ms}$ were removed (.20\% of trials in the typically developing group and $.26 \%$ of trials in the autism group). Trials where no response was made within $2500 \mathrm{~ms}$ were modelled as non-terminating accumulation trajectories, where the probability of a non-response occurring was the survivor function for the model at the time of the $2500 \mathrm{~ms}$ deadline $\mathrm{e}^{50-52}$. These trials accounted for $1.01 \%$ of the data in the typical group and $.95 \%$ of the data in the autism group. We fit the data from each task with hierarchical, Bayesian diffusion models with 5 parameters: 1) average drift-rate across difficulty levels v.mean, 2) boundary separation $a, 3$ ) non-decision time ter, 4) difference in mean drift-rate between difficulty levels v.diff, and 5) starting point $z$. The stochastic noise in the model (s) was fixed at 0.1 to solve a scaling problem within the model, following convention ${ }^{12}$. There were 3 hyperparameters for each parameter reflecting the mean $(\mu)$ and standard deviation $(\sigma)$ across the two groups and the difference between groups $(\delta)$. This parameterization meant we 
could explicitly set priors on the differences between groups, which relates to our main hypotheses. Specifically, the priors were:

Data level:

$$
y_{p i} \sim \operatorname{diffusion}\left(a_{p}, z_{p}, \operatorname{Ter}_{p}, v_{p i}, s\right)
$$

Parameters:

$$
\begin{gathered}
a_{p} \sim N_{+}\left(\mu_{a} \pm \delta_{a}, \sigma_{a}\right) \\
z_{p} / a_{p} \sim T N_{0,1}\left(\mu_{z} \pm \delta_{z}, \sigma_{z}\right) \\
T e r_{p} \sim N_{+}\left(\mu_{T e r} \pm \delta_{T e r}, \sigma_{T e r}\right) \\
v_{p 1}-v_{p 2} \sim N\left(\mu_{v . \text { diff }} \pm \delta_{v . d i f f}, \sigma_{v . \text { diff }}\right) \\
\frac{v_{p 1}+v_{p 2}}{2} \sim N\left(\mu_{v . \text { mean }} \pm \delta_{v . \text { mean }}, \sigma_{v . \text { mean }}\right) \\
s=0.1
\end{gathered}
$$

Hyperparameters:

$$
\begin{gathered}
\mu_{a} \sim N_{+}(0.2,0.2) \\
\mu_{z} \sim T N_{0,1}(0.5,0.2) \\
\mu_{T e r} \sim N_{+}(0.3,0.3) \\
\mu_{v . \text { diff }} \sim N(0,0.1) \\
\mu_{v . \text { mean }} \sim N(0.3,0.3) \\
\sigma_{a}, \sigma_{z}, \sigma_{T e r}, \sigma_{v . \text { diff }}, \sigma_{v . \text { mean }} \sim \Gamma(1,1) \\
\delta_{a}, \delta_{z}, \delta_{T e r}, \delta_{v . \text { diff }}, \delta_{v \text {.mean }} \sim N(0,0.01)
\end{gathered}
$$

where $y$ reflects the data, and subscripts $p$ and $i$ reflect the participant and difficulty level respectively. The priors for the $\mu$ and $\sigma$ parameters were based on previous studies applying hierarchical diffusion models ${ }^{53-55}$, and the priors for the $\delta$ parameters were derived from the "moderately informative priors" used for condition differences in [56]. We used a 
differential evolution Markov chain Monte Carlo algorithm (DE-MCMC ${ }^{57,58}$ ) to sample from the posterior with 15 interacting chains. Each chain had 4000 iterations, with the first 1500 discarded as burn-in. We also used a migration algorithm ${ }^{58}$, in which chains were randomly migrated every 14 iterations between iterations 500 and 1100 .

Bayesian t-tests using the BayesFactor $\mathrm{R}$ package ${ }^{59}$ showed that the final groups $(\mathrm{n}=$ 50 per group) were similar in age (Bayes factors $[\mathrm{BF}]$ in support of group differences $=0.25$ ), but there was inconclusive evidence $(1 / 3<\mathrm{BF}<3)$ for group differences in performance IQ $(\mathrm{BF}=1.12)$. The blind modeller therefore constructed models with and without the effects of performance IQ partialled out, so that we could assess the effect that this had on the results. The first author $(\mathrm{CM})$ then conducted the unblinded analysis with correct group membership. In light of a previous study finding reduced drift-rate in children with dyslexia ${ }^{34}$, we also assessed group differences in an exploratory model which partialled out reading and spelling ability.

\section{EEG preprocessing and analyses}

EEG data were band-pass filtered between 0.3 and $40 \mathrm{~Hz}$ using NetStation filters before being further processed in MATLAB with EEGLAB functions ${ }^{60}$. We downsampled each participant's data to $250 \mathrm{~Hz}$ and extracted only the data between the first fixation onset and the last offset period. We again bandpass-filtered between 0.3 and $40 \mathrm{~Hz}$ (due to insufficient attenuation of low frequencies by NetStation filters ${ }^{61}$ ) and used EEGLAB's clean_artifacts function to remove bad channels and identify data segments with standard deviations over 15 and correct them with artifact subspace reconstruction ${ }^{62}$. Missing channels were then interpolated. We then ran independent components analysis on $3000 \mathrm{~ms}$ epochs starting at fixation onset using an Infomax algorithm and subtracted ocular components from the continuous data. Finally we re-referenced the data to an average reference. 
We used a data-driven component decomposition technique, Reliable Components Analysis ${ }^{63,64}$, to identify spatiotemporally reliable patterns of activity across trials, for integration with our diffusion model. First, we epoched each participant's preprocessed continuous data from $600 \mathrm{~ms}$ before the response to $200 \mathrm{~ms}$ after the response, for all responses made between $200 \mathrm{~ms}$ and $2500 \mathrm{~ms}$ after stimulus onset. We baselined the data to the last $100 \mathrm{~ms}$ of the random motion period and submitted the baselined epochs for participants in both groups to Reliable Components analysis for each task separately. The forward-model projection of the weights for the most reliable component for each task (which explained $33.7 \%$ and $30.5 \%$ of the reliability in the motion coherence and direction integration tasks, respectively) is shown in Figure 3. This component resembled the most reliable component found in our previous work ${ }^{33,34}$, which resembles the centro-parietal positivity $^{65,66}$ and has been linked to drift-rate in typically developing children ${ }^{33}$ and children with dyslexia ${ }^{34}$. To investigate links with drift-rate in this dataset, we multiplied each participant's continuous data with the spatial weights for this component to yield a single component average waveform for each participant.

In our paradigm, stimulus-locked and response-locked activity will overlap temporally, especially for short response times. Therefore, in order to extract a responselocked measure for inclusion in the diffusion model, we used a linear deconvolution method to unmix the overlapping stimulus-locked and response-locked activity using the Unfold toolbox ${ }^{67}$. We modelled the projected continuous data for each participant by selecting a time window from $1000 \mathrm{~ms}$ before to $1000 \mathrm{~ms}$ after each stimulus event or response event made between $200 \mathrm{~ms}$ and $2500 \mathrm{~ms}$ following stimulus onset. We specified a design matrix with predictors for each difficulty level (difficult, easy) and each event type (stimulus, response). Next we time-expanded the design matrix by adding a predictor for each timepoint sampled for each event type and excluded segments with amplitudes above $250 \mathrm{mV}$ from the design 
matrix (motion coherence task: mean $2.72 \%$ of the data for each participant, range: 0 43.49\%; direction integration task: mean $=3.00 \%$, range: $0-49.30 \%)$. We then fit the deconvolution model resulting in regression weights (betas) for each of the 2 difficulty levels, 2 event types and 500 timepoints, which we used to construct regression ERP waveforms. The response-locked waveforms are presented in Figure 3 and stimulus-locked waveforms are presented in Supplementary Figure S1. Additionally, as a complement to previous analyses $^{35}$, Supplementary Figure S2 shows stimulus-locked waveforms relating to the second-most reliable component, previously linked to motion-specific processing ${ }^{35}$. While not the focus of the current investigation, this figure suggests that the groups were similar in their early encoding of motion evidence (up to $~ 400 \mathrm{~ms}$ following stimulus onset, see also [35]).

While the non-deconvolved waveforms showed the expected amplitude differences between the two difficulty levels, these differences were not apparent in the deconvolved waveforms. As the difference between difficulty levels changed following deconvolution, we considered the possibility that the overlap between stimulus- and response-locked activity differed between the difficulty levels, due to different RT distributions in each difficulty level. However, the non-deconvolved waveforms showed a difference between difficulty levels even when the RT distributions were matched across the two levels, suggesting that such differences cannot be solely attributed to different RT distributions. Instead, we suspected that the beta estimates were noisy and that the deconvolution technique was overfitting the noise. Therefore, in the final step where we extracted EEG measures for inclusion in the diffusion model, we re-ran the deconvolution model using a ridge-regression regularisation method which penalises the squared magnitude of the regression coefficients (see [68]) to minimise noise. Accordingly, the difficulty level differences were preserved when applying deconvolution with ridge-regression regularisation. We found the best 
regularisation parameter for each participant using cross-validation, and then took the mode across all participants and constrained the regularisation parameter to ensure that differences in regularisation did not contribute to group differences in resulting waveforms. The modal parameter value was 4.5 for the motion coherence task (5.5 and 6 for the autistic and typically developing children, separately) and 5.5 for the direction integration task (5.5 in both groups separately). We then fit a linear regression slope to each participant's average deconvolved waveform for each difficulty level from $200 \mathrm{~ms}$ before the response to the time of the response, to obtain a slope measure which we entered into the joint diffusion model and related to drift-rate. We investigated group differences in this slope measure using a Bayesian repeated measures ANOVA using $\mathrm{JASP}^{69}$, with difficulty level as a within-participants factor and group as a between-participants factor.
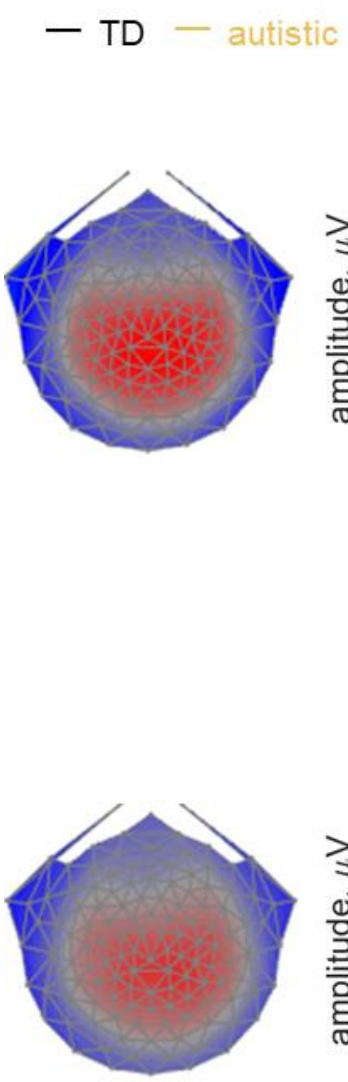

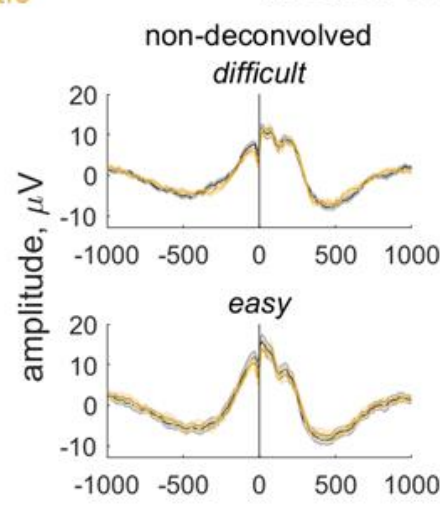

motion coherence task: response-locked
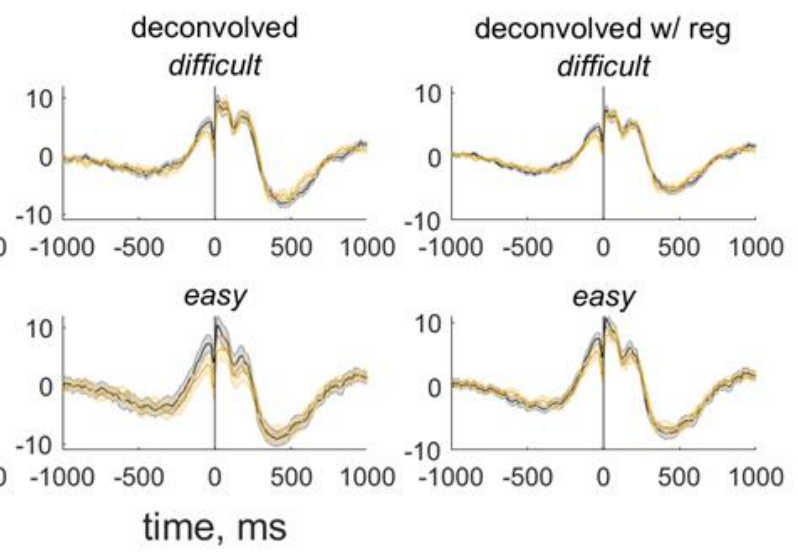

, ms
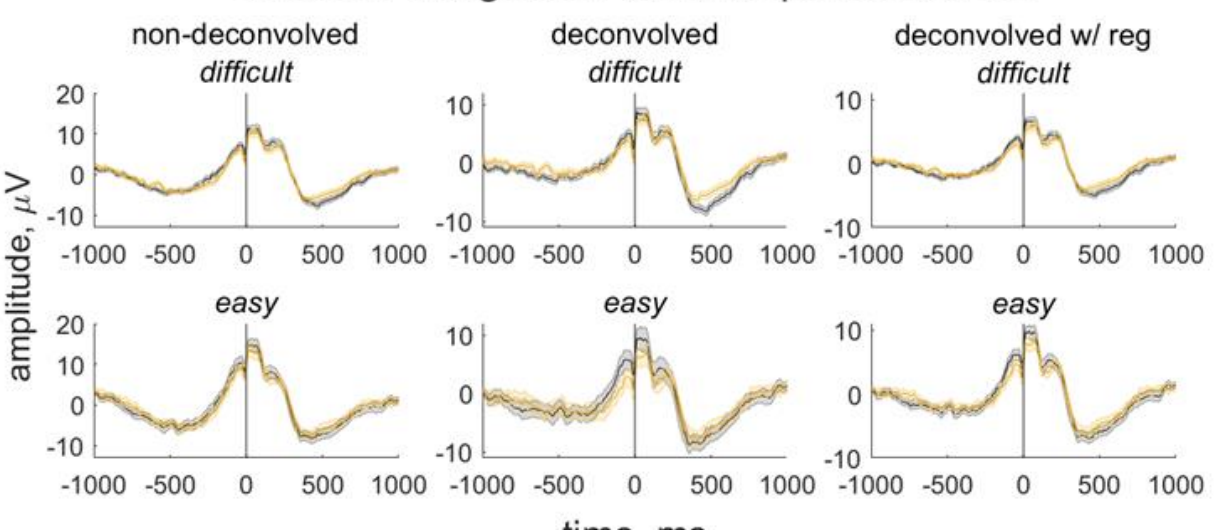

time, ms

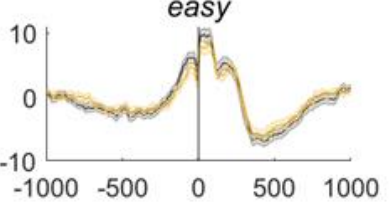


Figure 3. Response-locked evoked potentials for the motion coherence task and direction integration task

Topographic maps represent the forward-model projections of the most reliable component reflecting the weights given to each electrode following reliable components analysis (RCA) on data from all participants pooled across difficulty level for the motion coherence task (top) and direction integration task (bottom). Each individual's continuous data were multiplied by these weights to provide a component average waveform, with group average response-locked waveforms ( $\pm 1 \mathrm{SEM})$ shown for typically developing children (TD; grey) and autistic children (orange) for difficult and easy levels. The left column shows nondeconvolved group average waveforms. The central column shows deconvolved group average waveforms (without regularisation). The right column shows deconvolved group average waveforms with regularisation (ridge regression).

\section{Joint modelling: EEG}

To assess the relationship between drift-rate and the EEG measure discussed above, we used a joint modelling approach ${ }^{50,70-73}$. We estimated additional hyper-parameters for the correlation between the v.mean parameter and the average of the EEG measure over difficulty levels (EEG.mean), and between the v.diff parameter and the difference in the EEG measure between difficulty levels (EEG.diff). This meant that the structure of the original hierarchical model (with age partialled out) only differed for the drift-rate parameter, which was now a bivariate normal with the EEG measure:

$$
\begin{gathered}
{\left[v_{p 1}-v_{p 2}, E E G_{p 1}-E E G_{p 2}\right] \sim} \\
B N\left(\left[\mu_{v . d i f f} \pm \delta_{v . d i f f}, \mu_{E E G . d i f f} \pm \delta_{E E G . d i f f}\right],\left[\sigma_{v . d i f f}^{2}, \sigma_{v . d i f f} \sigma_{E E G . d i f f} \boldsymbol{\rho}, \sigma_{E E G . d i f f} \sigma_{v . d i f f} \boldsymbol{\rho}, \sigma_{E E G . d i f f}^{2}\right]\right) \\
{\left[\left(v_{p 1}+v_{p 2}\right) / 2,\left(E E G_{p 1}+E E G_{p 2}\right) / 2\right] \sim} \\
B N\left(\left[\mu_{v . m e a n} \pm \delta_{v . m e a n}, \mu_{E E G . m e a n} \pm \delta_{E E G . m e a n}\right],\left[\sigma_{v . m e a n}^{2}, \sigma_{v . m e a n} \sigma_{E E G . m e a n} \boldsymbol{\rho}, \sigma_{E E G . m e a n} \sigma_{v . m e a n} \boldsymbol{\rho}, \sigma_{E E G . m e a n}^{2}\right]\right) \\
\mu_{E E G . \text { diff }} \sim N(0,0.5) \\
\mu_{E E G . m e a n} \sim N(0,1) \\
\sigma_{E E G . d i f f}, \sigma_{E E G . m e a n} \sim \Gamma(1,1) \\
\delta_{E E G . d i f f}, \delta_{E E G . m e a n} \sim N(0,0.01) \\
\boldsymbol{\rho} \sim U(-1,1)
\end{gathered}
$$

where $\rho$ refers to the correlation between drift-rate and the EEG measure. We again used DE-

MCMC with 15 interacting chains to sample from the posterior of the joint model, though 
here we used only 3000 iterations, with the first 1000 discarded as burn-in and no migration algorithm, as the joint model was more computationally intensive. We estimated two variants of this joint model: one where the correlations were constrained to be the same across groups, which would allow for the estimation of more precise posteriors, and another less constrained version were the correlations were estimated separately for each group. While we did not find evidence for group differences in age, we partialled out age in this joint model, because both the EEG parameter and drift-rate are known to change with age ${ }^{33}$. This ensured that any correlations identified between the EEG parameter and drift-rate did not merely reflect agerelated changes.

\section{Joint modelling: ADHD}

As the two groups differed in terms of ADHD symptoms, and in recognition of the literature on decision-making differences in children with inattention and hyperactivity symptoms $\mathrm{s}^{20,45}$, we ran further joint models to investigate the relationship between levels of parent-reported ADHD symptoms (for inattention and hyperactivity/impulsivity domains) and diffusion model parameters. We ran separate models which each correlated parentreported ADHD symptoms with a different diffusion model parameter of interest, and we estimated the correlations separately for each group.

The priors for the ADHD joint model were near identical to the EEG joint model, though for the ADHD joint model (1) there were 5 different models, covering each parameter that could correlate with ADHD, (2) there were 2 ADHD subscales to assess (inatt, hyper), and (3) these subscales obviously did not vary between difficulty levels, meaning that there were no mean and difference values. The priors for the boundary separation-ADHD joint model can be seen below, with all other ADHD joint models following the same format: 


$$
\begin{gathered}
{[a, \text { inatt }] \sim B N\left(\left[\mu_{a} \pm \delta_{a}, \mu_{\text {inatt }} \pm \delta_{\text {inatt }}\right],\left[\sigma_{a}^{2}, \sigma_{a} \sigma_{\text {inatt }} \boldsymbol{\rho}, \sigma_{\text {inat }} \sigma_{a} \boldsymbol{\rho}, \sigma_{\text {inatt }}^{2}\right]\right)} \\
{[a, \text { hyper }] \sim B N\left(\left[\mu_{a} \pm \delta_{a}, \mu_{\text {hyper }} \pm \delta_{\text {hyper }}\right],\left[\sigma_{a}^{2}, \sigma_{a} \sigma_{\text {hyper }} \boldsymbol{\rho}, \sigma_{\text {hyper }} \sigma_{a} \boldsymbol{\rho}, \sigma_{\text {hyper }}^{2}\right]\right)} \\
\mu_{\text {inatt }} \sim N(0.5,0.2) \\
\mu_{\text {hyper }} \sim N(0.5,0.2) \\
\sigma_{\text {inatt }}, \sigma_{\text {hyper }} \sim \Gamma(1,1) \\
\delta_{\text {inatt }}, \delta_{\text {hyper }} \sim N(0,0.01) \\
\boldsymbol{\rho} \sim U(-1,1)
\end{gathered}
$$

\section{Results}

Accuracy and response time

The groups performed similarly in terms of overall accuracy and median RT, as shown in Figure 4, with considerable between-participants variability.
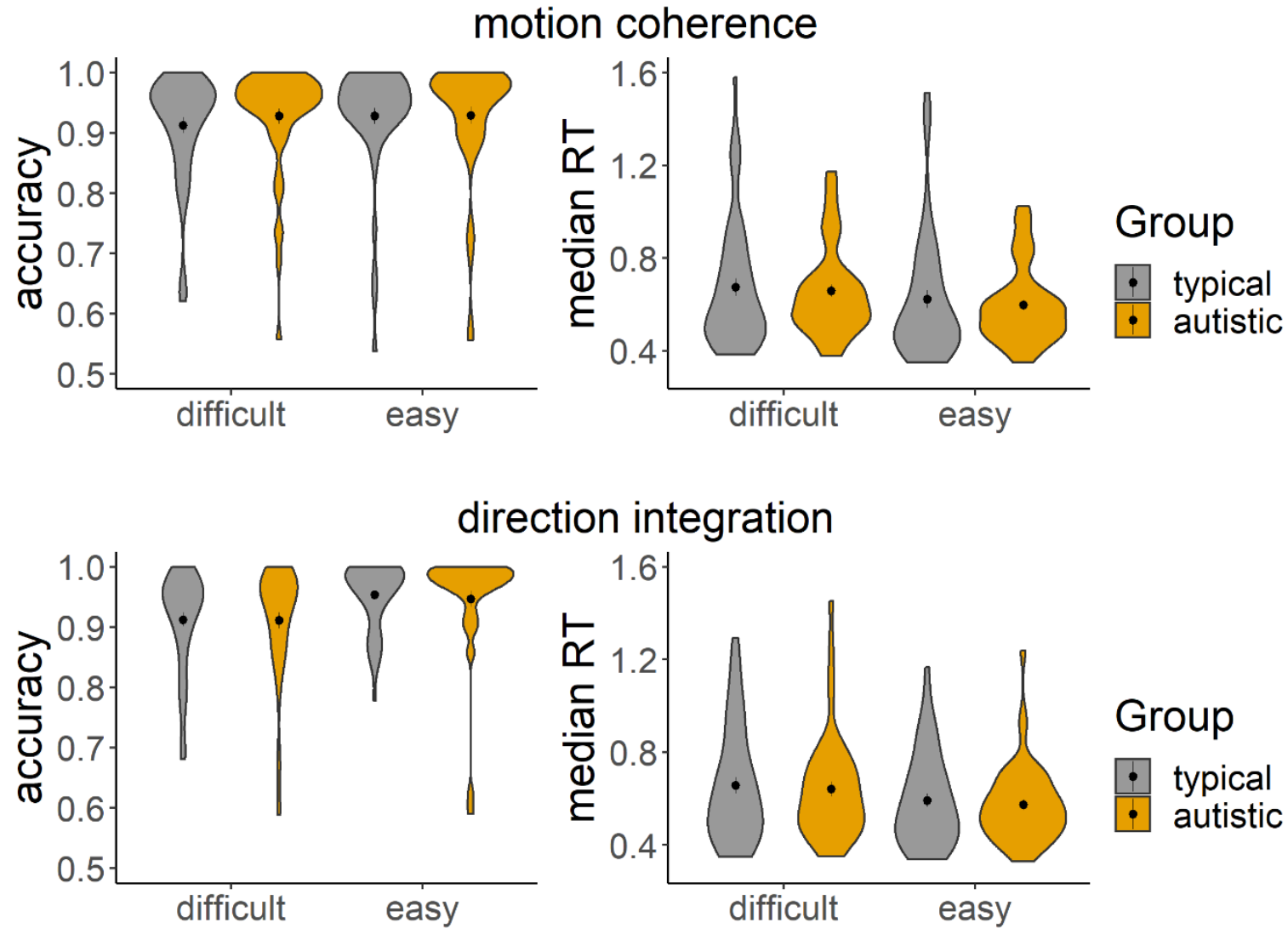

Figure 4. Accuracy and median response time (RT) for correct trials 
Violin plots showing the kernel probability density for each group's accuracy (left) and median RT (in seconds) for correct trials (right) for each difficulty level and each task (upper: motion coherence; lower: direction integration). Data for typically developing children and autistic children are presented in grey and orange, respectively. Dots and vertical lines represent the group mean and \pm 1 SEM.

\section{Diffusion modelling of behavioural data}

Gelman-Rubin diagnostic values ${ }^{74}$ for all chains in all models were under $1.1($ mean $=$ 1.00 , range: $1.00-1.09)$, reflecting good convergence. To check model fit, we plotted the defective cumulative density functions for each group (Supplementary Figure S3). Figure 5 shows the prior and posterior distributions for the group-level parameters that reflect the difference between groups for each of the 5 parameters (v.mean, a, ter, v.diff, z/a), along with Bayes factors calculated through the Savage-Dickey ratio. Bayes factors above 1 reflect more evidence for the alternative hypothesis of group differences compared to the null hypothesis, whereas Bayes factors below 1 reflect relatively more evidence for the null hypothesis than the alternative hypothesis. Bayes factors above 3 reflect 'substantial' or 'moderate' evidence for the alternative hypothesis, whereas Bayes factors below $1 / 3$ reflect 'substantial' or 'moderate' evidence in support of the null hypothesis ${ }^{75,76}$. 

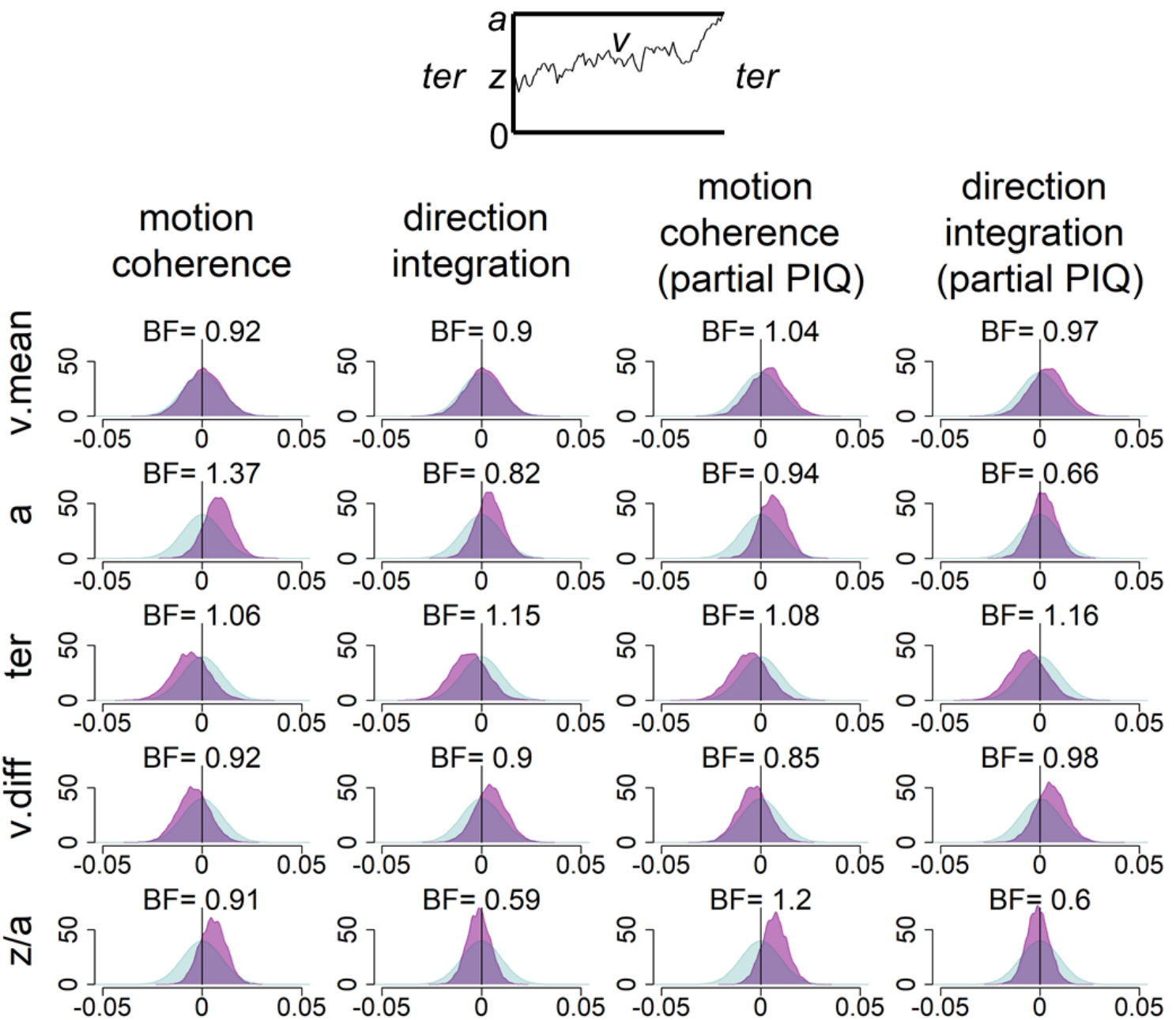

Figure 5. Prior and posterior density distributions

Prior (blue) and posterior (purple) density distributions for the group-level parameters reflecting group differences in each of the 5 model parameters $(v \cdot m e a n=$ mean drift rate across difficulty levels; $\mathrm{a}=$ boundary separation; ter = non-decision time; v.diff $=$ difference in mean drift rate between difficulty levels; $z / a=$ relative starting point) for each task. Inset is a schematic representation of the model parameters. Columns 1 and 2 show the results of the standard model and Columns 3 and 4 show the results of the model with performance IQ partialled out. Positive values reflect higher parameter values in the autistic group compared to the typically developing group. $\mathrm{BF}=$ Savage-Dickey Bayes factors in favour of the alternative hypothesis over the null hypothesis, where BF $>1$ reflect more support for the alternative hypothesis.

As shown in Figure 5, all Bayes factors were between 1/3 and 3, reflecting inconclusive evidence for our hypotheses. The posterior distributions were close to the prior distributions, suggesting that the data were relatively uninformative. Specifically, we had hypothesised that autistic children would have reduced drift rates in the motion coherence task and increased drift-rates in the direction integration task, compared to typically 
developing children. Figure 5 suggests that there was little difference between groups in mean drift-rate in the standard model (as the posterior distribution for v.mean is centred close to 0 ), with weak, anecdotal evidence in favour of the null hypothesis $(\mathrm{BF}=0.92$ and $\mathrm{BF}=0.9$ for the motion coherence and direction integration tasks, respectively). When partialling out performance IQ there were very small group differences reflecting increased drift-rate in autistic children in both tasks (shown by the small rightward shift in the posterior distribution for v.mean), but the Bayes factors were very close to $1(\mathrm{BF}=1.04$ and $\mathrm{BF}=0.97)$, suggesting equivocal evidence for the alternative and null hypotheses. We also hypothesised that autistic children would show wider response boundaries compared to typically developing children, and while the group difference was in the expected direction (shown by a rightward shift of the posterior distribution for $a$ ), the Bayes factors were again very close to 1 in the standard model $(\mathrm{BF}=1.37$ and $\mathrm{BF}=0.82)$, and the evidence in support of the alternative hypothesis reduced slightly when controlling for the effects of performance $\mathrm{IQ}(\mathrm{BF}=0.94$ and $\mathrm{BF}=$ 0.66). Finally, we hypothesised that autistic children would show longer non-decision times than typically developing children. Instead, the autistic children had slightly shorter nondecision times in both tasks (shown by a leftward shift of the posterior distribution for ter), but the Bayes factors were once again very close to 1 , both in the standard model $(\mathrm{BF}=1.06$, $\mathrm{BF}=1.15)$ and when controlling for performance $\mathrm{IQ}(\mathrm{BF}=1.08, \mathrm{BF}=1.16)$. These results suggest that more data would be required to provide conclusive evidence to support or refute our hypotheses.

In a related study of dyslexia, we found that children with dyslexia had reduced driftrates compared to typically developing children ${ }^{34}$. Given that the autistic children had lower reading and spelling abilities than typically developing children, overall (Table 1), we conducted a follow-up analysis to ensure that the pattern of results did not change when controlling for children's reading ability (assessed using the TOWRE-2 Phonemic Decoding 
Efficiency subscore) and spelling ability, in addition to performance IQ (see Supplementary Figure S4). While some of the Bayes factors increased slightly, there remained no conclusive evidence for any of our hypotheses, with all Bayes factors being between 1/3 and 3 .

\section{Joint model: $E E G$}

Figure 6 shows that autistic children had a slightly shallower build-up of activity in the centro-parietal component from $200 \mathrm{~ms}$ before the response to the point of the response compared to typically developing children. However, Bayesian ANOVAs revealed no evidence for group differences: the best model of the EEG slope data included only a main effect of difficulty level, for both tasks. When averaging across models, there was insufficient evidence in either task for including a main effect of group (motion coherence: $\mathrm{BF}=0.73$; direction integration: $\mathrm{BF}=0.34$ ) or an interaction between group and difficulty level (motion coherence: $\mathrm{BF}=0.63$; direction integration: $\mathrm{BF}=0.27$ ). Following Manning et al. ${ }^{33,34}$, we expected this EEG measure to relate to drift-rate in a joint model. Supplementary Figure S5 plots individual estimates of drift-rate against the EEG measure entered into the joint model. 

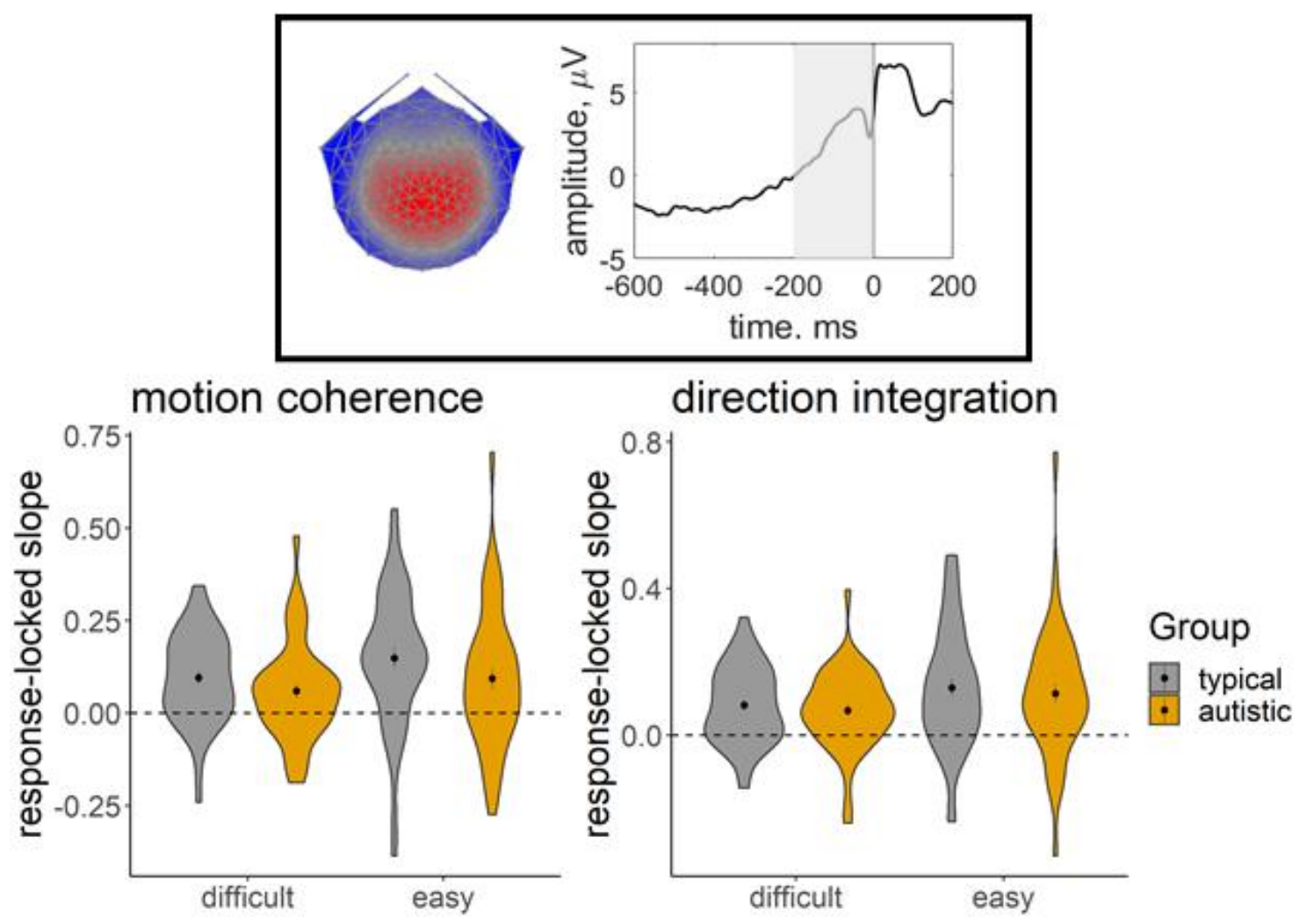

Figure 6. EEG slope measure extracted for inclusion in the joint model

Violin plots showing the kernel probability density for the EEG slope measure extracted for inclusion in the joint model for each group (typically developing: grey; autistic: orange) for each difficulty level. The extracted measure was the slope of a linear regression line fitted to each participant's deconvolved (with regularisation) response-locked waveform, from $200 \mathrm{~ms}$ prior to the response to the response (see shaded area of schematic responselocked waveform in inset). The dotted line reflects a flat slope. Dots and vertical lines represent the group mean and \pm 1 SEM.

We first fit a joint model where a single correlation between EEG and drift-rate was estimated across all participants, for each task (Figure 7, blue distribution). There was weak evidence for a positive correlation between the mean EEG measure across difficulty levels and the mean drift-rate across difficulty levels in the motion coherence task (posterior mean correlation $=.23,95 \%$ credible intervals $=[.02, .42], \mathrm{BF}=1.31)$. The correlation was weaker in the direction integration task, with credible intervals encompassing 0 and $\mathrm{BF}<1$ (posterior mean $=.18,95 \% \mathrm{CI}=[-.03, .38], \mathrm{BF}=.56)$. The difference in drift-rate between difficulty levels did not clearly relate to the difference in EEG measure between difficulty levels 
(motion coherence task: posterior mean $=-.04,95 \% \mathrm{CI}=[-.28, .20], \mathrm{BF}=.17$; direction integration task: posterior mean $=.06,95 \% \mathrm{CI}=[-.19, .30], \mathrm{BF}=.17)$.
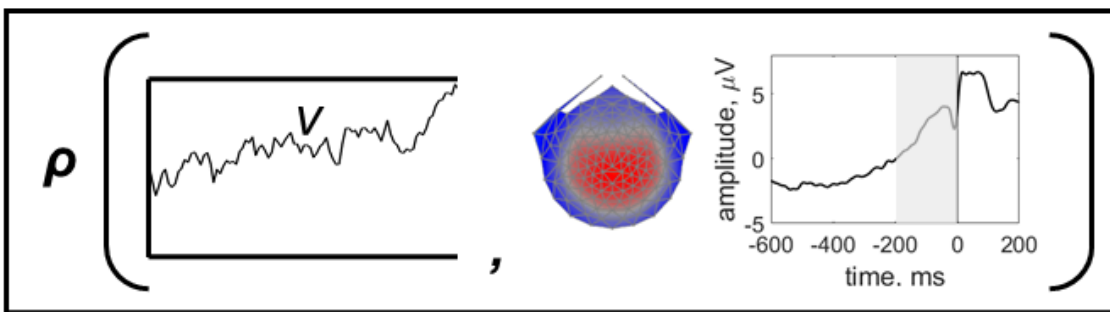

motion coherence mean

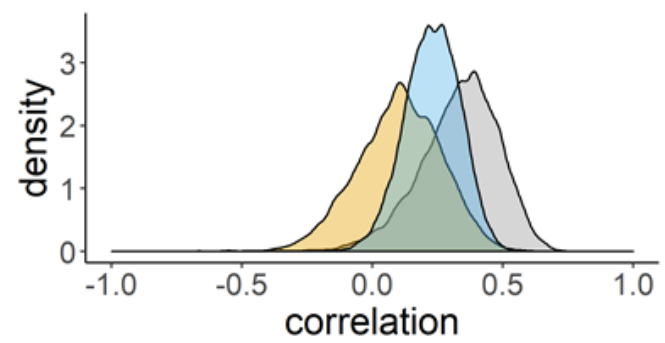

direction integration

mean

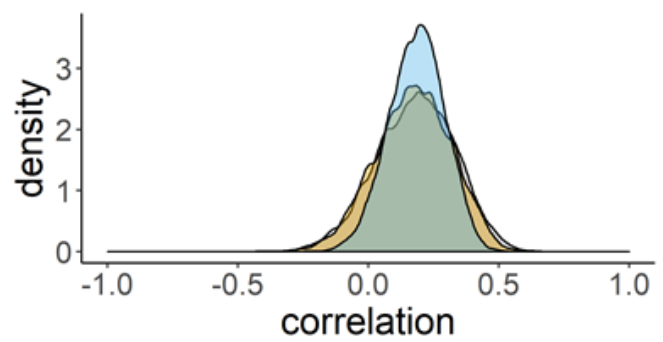

motion coherence difference

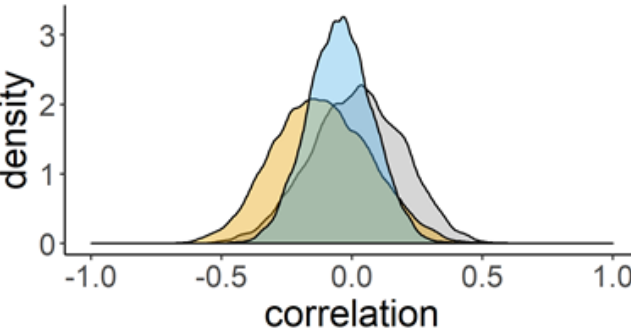

direction integration difference

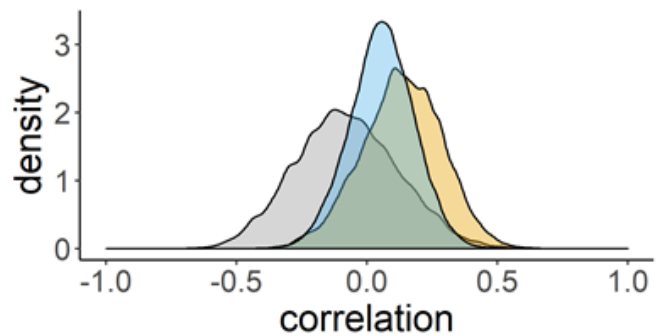

\section{Group}

$\square$ typical all

Figure 7. Posterior density plots showing the correlation between drift-rate and the EEG measure

Inset provides a schematic representation of the drift-rate parameter ( $v$; left) and EEG measure (slope of response-locked waveform from $-200 \mathrm{~ms}$ to $0 \mathrm{~ms}$ around the response; right) that were correlated in the joint model, where $\rho$ represents the correlation. Posterior density plots in the left column reflect the correlation between the mean drift-rate across difficulty levels (v.mean) and the mean EEG slope measure across difficulty levels (EEG.mean). Posterior density plots in the right column reflect the correlation between the difference in drift-rate between difficulty levels (v.diff) and the difference in EEG slope measure between difficulty levels (EEG.diff). Plots for the motion coherence task are presented in the upper row and plots for the direction integration task are presented in the lower row. The blue distribution shows the correlation across all participants, and the grey and orange distributions show separate correlations estimated for typically developing children and autistic children, respectively.

Next we ran joint models where a separate correlation coefficient between EEG and drift-rate was estimated for each group (Figure 7, grey and orange distributions), in order to 
see if the relationship could be found in each group (rather than explicitly testing for differences in correlations between groups). In the motion coherence task, there was a positive relationship between mean drift-rate and the mean EEG measure across difficulty levels for the typically developing children (posterior mean $=.33,95 \% \mathrm{CI}=[-.01, .59], \mathrm{BF}=$ 1.64), but there was no clear correlation for the autistic children (posterior mean $=.10,95 \%$ $\mathrm{CI}=[-.22, .40])$ with moderate support $(\mathrm{BF}=.28)$ for the null hypothesis of no relationship. Moreover, there was no correlation between difference in drift-rate and difference in EEG measure between difficulty levels in the motion coherence task, for either group (typically developing: posterior mean $=.02,95 \% \mathrm{CI}=[-.33, .35], \mathrm{BF}=.23$; autistic: posterior mean $=-$ $.12,95 \% \mathrm{CI}=[-.46, .25], \mathrm{BF}=.31)$.

In the direction integration task, there was a small positive correlation between mean drift-rate and mean EEG measure across difficulty levels in both groups, but the evidence for this relationship was inconclusive (typically developing: posterior mean $=.18,95 \% \mathrm{CI}=[-$ $.13, .46], \mathrm{BF}=.41$; autistic: posterior mean $=.17,95 \% \mathrm{CI}=[-.12, .44], \mathrm{BF}=.35)$. There was no evidence for a correlation between difference in drift-rate and difference in EEG measure between difficulty levels, for either group (typically developing: posterior mean $=-.08,95 \%$ $\mathrm{CI}=[-.44, .31], \mathrm{BF}=.28$; autistic: posterior mean $=.13,95 \% \mathrm{CI}=[-.18, .42], \mathrm{BF}=.30)$.

\section{Joint model: $A D H D$}

Finally, we investigated whether diffusion model parameters were related to parentreported ADHD symptoms, in a series of joint models estimating a separate correlation for each group, with each model allowing inattentiveness and hyperactivity/impulsivity to relate to a different model parameter of interest (v.mean, a, ter). Scatterplots showing the relationship between ADHD dimensions and parameter estimates from the basic model are provided in Supplementary Figures S6 and S7. The posterior distributions for the correlations 
arising from the joint models are presented in Supplementary Figures S8-S10. In the autism group only, we found weak evidence for a negative relationship between mean drift-rate and hyperactivity/impulsivity for both tasks (motion coherence task: posterior mean $=-.31,95 \%$ $\mathrm{CI}=[-.54,-.03], \mathrm{BF}=1.82$; direction integration task: posterior mean $=-.27,95 \% \mathrm{CI}=[-.51$, $0], \mathrm{BF}=1.27$; Figure $\mathrm{S} 8$ ). For all other relationships, there was relatively more evidence in favour of the null hypothesis (BFs $<1$, see Table $\mathrm{S} 1$ ).

\section{Discussion}

In this pre-registered study with a blind modelling approach, we presented autistic and typically developing children with two motion processing tasks that have previously been linked to reduced or increased sensitivity in autism ${ }^{22-24,32}$. To our knowledge, for the first time in the context of autism, we used a combination of approaches that are sensitive to different stages of processing: diffusion modelling and EEG. While the autistic children had slightly increased boundary separation (reflecting increased response caution) compared to typically developing children, the evidence for or against group differences was inconclusive. Moreover, we did not find conclusive evidence to support or refute the hypothesised group differences in evidence accumulation (drift-rate) and non-decision time. We identified a response-locked centro-parietal EEG component, but build-up in this EEG measure was not consistently related to drift-rate in the autism group. There was weak evidence for a relationship between parent-report measures of ADHD and drift-rate in the autism group, with higher levels of hyperactivity/impulsivity being associated with a lower drift-rate, in both tasks.

First we consider why we did not find evidence for the expected group differences in drift-rate in the two tasks. The autistic children were slightly more accurate and slightly faster than the typically developing children in both tasks, and this was reflected in the autistic 
children having slightly increased drift-rates compared to typically developing children (when controlling for performance IQ), although the evidence was inconclusive, with Bayes factors very close to 1 . The small difference between groups was in the direction of our hypothesis for the direction integration task, for which enhanced performance has been previously reported in autistic children of a similar age $\mathrm{e}^{22}$. Yet, the result was not in the hypothesised direction for the motion coherence task, for which reduced sensitivity has been reported in autistic individuals (see [26] for meta-analysis). We note that reduced performance in motion coherence tasks is not consistently found in the literature e.g., 22,77,78, but we know of no reports of enhanced sensitivity. While a range of stimulus parameters could contribute to discrepant results, we think the most important reason that we did not find reduced sensitivity here was because we were not assessing performance at threshold, unlike previous studies assessing motion coherence thresholds in autism ${ }^{\text {e.g., } 23,24,32}$. In the current study, we chose to focus on just two coherence levels, which were selected through piloting to ensure that they would be difficult enough to obtain errors in even the oldest children, as required for diffusion modelling, but easy enough that even the youngest children could do the task. Accordingly, the data were well fit by our diffusion models. Yet, if the main difficulty faced by autistic children during motion coherence tasks is filtering out the randomly moving noise dots ${ }^{22,30}$, it is conceivable that autistic children may respond similarly, or even with greater sensitivity, relative to typically developing children, at high coherence levels. Future studies could increase the number and range of coherence levels, to investigate whether group differences would emerge at lower levels of coherence.

While group differences in drift-rate are likely to be task dependent, the existing literature has shown relatively consistent differences between autistic and non-autistic participants in boundary separation ${ }^{17-21}$ (but see [79], which appears to find little difference in boundary separation between groups for a perceptual task). Our results are in the 
hypothesised direction, with autistic children having slightly wider boundary separation than typically developing children on average. However, this evidence was inconclusive, with relatively more evidence in favour of the null hypothesis than the alternative hypothesis of group differences in both tasks when controlling for performance IQ. This discrepancy with the literature could reflect differences between tasks, or even task instructions. In our paradigm, we explicitly asked participants to emphasise speed and accuracy, and awarded points based on both (i.e., an efficiency score), which differs from other studies that reported group differences in boundary separation. For example, in their study of autistic children, Pirrone et al. ${ }^{18}$ did not appear to instruct participants to emphasise response speed, and no feedback was provided. Therefore, it is conceivable that autistic children have a tendency to emphasise accuracy over speed, but when they are asked to emphasise both speed and accuracy, they are able to modulate their boundary separation to some extent. Future research would be required to further ascertain the effects of instructions on boundary separation in autistic participants, as previously investigated in individuals with $\mathrm{ADHD}^{80}$.

We originally hypothesised that autistic children would show increased non-decision time compared to typically developing children, based on Pirrone et al. ${ }^{17}$, but note that more recent research suggests no differences between autistic and non-autistic children in this parameter ${ }^{18}$. In the current study, there was slightly more evidence in favour of the alternative hypothesis of group differences compared to the null hypothesis, with autistic children having slightly shorter non-decision times. As non-decision times encompass both sensory encoding and response generation processes, we might expect non-decision time estimates to vary according to the type of stimulus to be encoded (e.g., a single grating in Pirrone et al. ${ }^{18}$ vs. multiple white dots in the current study). Future work will therefore be required to understand whether pre-decision (sensory encoding) and post-decision (response generation) processes differ in autistic and non-autistic participants, for a range of stimuli. 
While we have discussed the evidence for each of our hypotheses separately, we note that many of our Bayes factors were close to 1. As the prior and posterior distributions are similar, the data appear to be relatively uninformative, suggesting that any group differences are small. One factor potentially contributing to the inconclusive evidence obtained across all of our hypotheses, while other studies have reached firmer conclusions, is that we used a conservative method of testing our hypotheses, which takes account of uncertainty in individual participant-level parameters. Instead, other studies e.g., 18,20 conducted inferential statistics on point estimates extracted from the diffusion model fitting procedure, thus not accounting for uncertainty in individual-level parameters, and potentially erroneously inflating the evidence in favour of the winning model ${ }^{81,82}$. In order to overcome the uncertainty associated with individual parameter estimates and provide stronger conclusions, we would need to increase the number of trials collected for each participant (which is challenging when working with children) and/or increase the sample of participants tested. While our study does not provide conclusive evidence to support or refute hypotheses regarding decision-making parameters in autistic children, looking ahead, the data from this study could be combined with that from previous studies to inform testing priors for further studies using diffusion models with autistic participants. It might also be informative to reanalyse the data from previous studies using a consistent analytical framework that takes into account the uncertainty of parameter estimates at the individual level, to see what effect this has.

To complement our diffusion modelling approach, we collected high-density EEG during task performance from the majority of participants. We identified a response-locked reliable centroparietal component resembling a component previously related to drift-rate in typically developing children ${ }^{33}$ and children with dyslexia ${ }^{34}$. We used a linear deconvolution method to unmix the overlapping stimulus-locked and response-locked activity and then 
extracted the steepness of the deconvolved response-locked waveform preceding the response to add to a joint model. Although we have previously found that the slope of this component relates to drift-rate in typically developing children and children with dyslexia in a motion coherence task, here joint modelling showed no evidence of a relationship between the slope of this component and drift-rate estimates in autistic children in the motion coherence task.

This result is intriguing as it demonstrates that there is not necessarily a 1:1 correspondence between the positive centro-parietal component and drift-rate. It has been shown that the centro-parietal positivity also reflects the subjective clarity of perceptual experience ${ }^{83}$, which likely relates to decision confidence. Accordingly, in the monkey brain, parietal neurons involved in decision-making also encode choice confidence ${ }^{84}$, and in humans, the same neural sources in prefrontal cortex and parietal cortex appear to be involved in evidence accumulation and confidence ${ }^{85}$. Atypical confidence judgments have been reported in autistic participants ${ }^{86-88}$, so future research will be required to investigate how confidence relates to our EEG component in autistic and typically developing children.

Another potential reason why there might not be a 1:1 relationship between our EEG component and drift-rate is that, in contrast to the assumption of sequential stages made by the diffusion model, early sensory encoding, evidence accumulation and motor planning may overlap ('continuous flow' ${ }^{94,89,90}$ ). If this is the case, the EEG component we identified might reflect more than just evidence accumulation, so that any differences in early sensory encoding and / or motor planning in autistic participants might affect the EEG component while the drift-rate is similar to non-autistic participants: a speculation requiring further investigation. While not the focus of the current investigation, we also note that the early stimulus-locked waveforms (up to $400 \mathrm{~ms}$ following stimulus onset) for autistic and typically developing children look very similar (see also [35]), suggesting that early encoding of evidence (i.e., the input to the decision-making stage) is also not disrupted in autistic 
children, so future studies could focus on motor planning as an alternative / additional reason why the typical relationship between EEG and drift rate in autistic children might not hold.

An outstanding issue is the considerable variability in participants' accuracy and response times, and resulting diffusion model parameter estimates. Similarly, substantial variability has been found previously in a typically developing sample ${ }^{33}$, even within children of the same age group. While some of this variability in estimates may be attributable to measurement error, the current study suggests at least one area of individual differences relating to variability in task performance: symptoms related to ADHD. In the autism group, there was weak evidence for a relationship whereby participants with high levels of hyperactivity had lower drift-rates. This result is in line with previous studies showing that individuals with ADHD had lower drift-rates than control participants in a range of tasks ${ }^{20,45,91-93}$. We did not find evidence for a relationship between ADHD symptoms and drift-rate in the typically developing sample, which we suspect is due to a lack of variance within the typically developing group on this measure. Future research could investigate whether this is also the case when using a measure that is designed to better capture variability along a continuum (e.g., Strengths and Weaknesses of ADHD-symptoms and Normal-behavior ${ }^{94}$ ). In light of our findings, we suggest that future diffusion model studies of autism should consider the moderating effects of co-occurring traits such as ADHD, and investigate which other factors might contribute to variability in parameter estimates.

In the same vein, it is informative to consider the current results in conjunction with a related study of children with dyslexia ${ }^{34}$. Children with dyslexia showed reduced drift-rates in both tasks: a pattern not seen in autistic children here. This is interesting given that elevated motion coherence thresholds have been reported in both developmental conditions ${ }^{95}$ (see [28] for review), with a theory that this reflects an underlying vulnerability in the development of the dorsal stream that is involved in motion processing ${ }^{28}$. By combining the current study and 
that of Manning et al. ${ }^{34}$, we suggest that motion processing differences are less pronounced in autistic children than they are for children with dyslexia. Complementing this finding, recent meta-analyses suggested a smaller effect size for coherent motion processing differences in autism $^{26}$ than in dyslexia ${ }^{96}$. There seem to be some similarities in how autistic children and children with dyslexia process motion information. For example, both autistic and dyslexic children differ from typically developing children at $~ 430 \mathrm{~ms}$ following stimulus onset in an occipital component specifically in the motion coherence task, but not the direction integration task $\mathrm{k}^{35}$, and both groups of children appear to show slightly reduced amplitudes in the response-locked centroparietal component, as shown in the current study and that of Manning et al. ${ }^{34}$. Yet, the pattern of behavioural performance, diffusion model parameters and relationship between model parameters and EEG differs in the two developmental conditions. Extending this paradigm may help to similarly identify areas of convergence and divergence between other developmental conditions, while also providing a framework to help link brain and behaviour. An additional benefit of the diffusion modelling approach is that sensitivity to sensory information can be compared between developmental conditions while taking account of potential differences in speed-accuracy tradeoffs.

\section{Data and Availability}

Analysis scripts and output files are available at: https://osf.io/f3wnt/. Data will be made available on the UK Data Service on acceptance.

\section{References}

1. American Psychiatric Association. Diagnostic and Statistical Manual, 5th edition (DSM5). (American Psychiatric Association, 2013). 
2. Bogdashina, O. Sensory perceptual issues in autism and Asperger syndrome: Different sensory experiences - different perceptual worlds. (Jessica Kingsley Publishers, 2003).

3. Jones, R. S. P., Quigney, C. \& Huws, J. C. First-hand accounts of sensory perceptual experiences in autism: a qualitative analysis. J. Intellect. Dev. Disabil. 28, 112-121 (2003).

4. Robertson, A. E. \& Simmons, D. R. The sensory experiences of adults with Autism Spectrum Disorder: A qualitative analysis. Perception 44, 569-586 (2015).

5. Robertson, C. E. \& Baron-Cohen, S. Sensory perception in autism. Nat. Rev. Neurosci. 18, 671-684 (2017).

6. Franklin, A. et al. Reduced chromatic discrimination in children with autism spectrum disorders. Dev. Sci. 13, 188-200 (2010).

7. Kwakye, L., Foss-Feig, J., Cascio, C., Stone, W. \& Wallace, M. Altered auditory and multisensory temporal processing in Autism Spectrum Disorders. Front. Integr. Neurosci. 4, 129 (2011).

8. Puts, N. A. J., Wodka, E. L., Tommerdahl, M., Mostofsky, S. H. \& Edden, R. A. E. Impaired tactile processing in children with autism spectrum disorder. J. Neurophysiol. 111, 1803-1811 (2014).

9. Bonnel, A. et al. Enhanced pitch sensitivity in individuals with autism: A signal detection analysis. J. Cogn. Neurosci. 15, 226-235 (2003).

10. Blakemore, S.-J. et al. Tactile sensitivity in Asperger syndrome. Brain Cogn. 61, 513 (2006).

11. Dickinson, A., Bruyns-Haylett, M., Smith, R., Jones, M. \& Milne, E. Superior orientation discrimination and increased peak gamma frequency in autism spectrum conditions. J. Abnorm. Psychol. 125, 412-422 (2016).

12. Ratcliff, R. A theory of memory retrieval. Psychol. Rev. 85, 59-108 (1978). 
13. Stone, M. Models for choice-reaction time. Psychometrika 25, 251-260 (1960).

14. Evans, N. J. \& Wagenmakers, E.-J. Evidence accumulation models: Current limitations and future directions. Quant. Methods Psychol. 16, 73-90 (2020).

15. Stafford, T., Pirrone, A., Croucher, M. \& Krystalli, A. Quantifying the benefits of using decision models with response time and accuracy data. Behav. Res. Methods 52, $2142-2155$ (2020).

16. White, C. N., Ratcliff, R., Vasey, M. W. \& McKoon, G. Using diffusion models to understand clinical disorders. J. Math. Psychol. 54, 39-52 (2010).

17. Pirrone, A., Dickinson, A., Gomez, R., Stafford, T. \& Milne, E. Understanding perceptual judgment in autism spectrum disorder using the drift diffusion model. Neuropsychology 31, 173-180 (2017).

18. Pirrone, A., Johnson, I., Stafford, T. \& Milne, E. A diffusion model decomposition of orientation discrimination in children with Autism Spectrum Disorder (ASD). Eur. J. Dev. Psychol. 17, 213-230 (2020).

19. Kirchner, J. C., Schmitz, F. \& Dziobek, I. Brief Report: Stereotypes in autism revisited. J. Autism Dev. Disord. 42, 2246-2251 (2012).

20. Karalunas, S. L. et al. Overlapping and distinct cognitive impairments in AttentionDeficit/Hyperactivity and Autism Spectrum Disorder without intellectual disability. $J$. Abnorm. Child Psychol. 46, 1705-1716 (2018).

21. Iuculano, T. et al. Neural correlates of cognitive variability in childhood autism and relation to heterogeneity in decision-making dynamics. Dev. Cogn. Neurosci. 42, 100754 (2020).

22. Manning, C., Tibber, M. S., Charman, T., Dakin, S. C. \& Pellicano, E. Enhanced integration of motion information in children with autism. J. Neurosci. 35, 6979-6986 (2015). 
23. Milne, E. et al. High motion coherence thresholds in children with autism. J. Child Psychol. Psychiatry 43, 255-263 (2002).

24. Manning, C., Charman, T. \& Pellicano, E. Processing slow and fast motion in children with Autism Spectrum Conditions. Autism Res. 6, 531-541 (2013).

25. Bridwell, D. A. et al. Moving beyond ERP components: A selective review of approaches to integrate EEG and behavior. Front. Hum. Neurosci. 12, 106 (2018).

26. Van der Hallen, R., Manning, C., Evers, K. \& Wagemans, J. Global motion perception in Autism Spectrum Disorder: A meta-analysis. J. Autism Dev. Disord. 49, 4901-4918 (2019).

27. Manning, C., Tibber, M. S. \& Dakin, S. C. Visual integration of direction and orientation information in autistic children. Autism Dev. Lang. Impair. 2, 2396941517694626 (2017).

28. Braddick, O., Atkinson, J. \& Wattam-Bell, J. Normal and anomalous development of visual motion processing: motion coherence and 'dorsal-stream vulnerability'. Neuropsychologia 41, 1769-1784 (2003).

29. Frith, U. \& Happé, F. Autism: beyond "theory of mind". Cognition 50, 115-132 (1994).

30. Zaidel, A., Goin-Kochel, R. P. \& Angelaki, D. E. Self-motion perception in autism is compromised by visual noise but integrated optimally across multiple senses. Proc. Natl. Acad. Sci. 112, 6461-6466 (2015).

31. Van de Cruys, S., Van der Hallen, R. \& Wagemans, J. Disentangling signal and noise in autism spectrum disorder. Brain Cogn. 112, 78-83 (2017).

32. Pellicano, E., Gibson, L., Maybery, M., Durkin, K. \& Badcock, D. R. Abnormal global processing along the dorsal visual pathway in autism: a possible mechanism for weak visuospatial coherence? Neuropsychologia 43, 1044-1053 (2005). 
33. Manning, C., Wagenmakers, E.-J., Norcia, A. M., Scerif, G. \& Boehm, U. Perceptual decision-making in children: Age-related differences and EEG correlates. Comput. Brain Behav. 4, 53-69 (2021).

34. Manning, C. et al. Visual motion and decision-making in dyslexia: Evidence of reduced accumulation of sensory evidence and related neural dynamics. 2021.05.26.21257878 https://www.medrxiv.org/content/10.1101/2021.05.26.21257878v1 (2021) doi:10.1101/2021.05.26.21257878.

35. Toffoli, L., Scerif, G., Snowling, M. J., Norcia, A. M. \& Manning, C. Global motion evoked potentials in autistic and dyslexic children: A cross-syndrome approach. Cortex 143, 109-126 (2021).

36. Dutilh, G. et al. A test of the diffusion model explanation for the worst performance rule using preregistration and blinding. Atten. Percept. Psychophys. 79, 713-725 (2017).

37. Schönbrodt, F. D. \& Wagenmakers, E.-J. Bayes Factor Design Analysis: Planning for compelling evidence. (2016).

38. Stuart, E. A., King, G., Imai, K. \& Ho, D. MatchIt: Nonparametric preprocessing for parametric causal inference. J. Stat. Softw. (2011) doi:10.18637/jss.v042.i08.

39. Wechsler, D. WASI-II: Wechsler abbreviated scale of intelligence. (PsychCorp, 2011).

40. Rutter, M., Bailey, A. \& Lord, C. Social Communication Questionnaire. (Western Psychological Services, 2003).

41. Lord, C., DiLavore, P. C. \& Gotham, K. Autism Diagnostic Observation Schedule, Second Edition (ADOS-2). (Western Psychological Services, 2012).

42. O’Brien, G. \& Yeatman, J. D. Bridging sensory and language theories of dyslexia: Toward a multifactorial model. Dev. Sci. 24, e13039 (2021). 
43. Torgesen, J. K., Wagner, R. K. \& Rashotte, C. A. Test of Word Reading Efficiency Second Edition (TOWRE-2). (Pro-Ed, 2012).

44. Wechsler, D. Wechsler individual achievement test - third UK edition (WIAT-III UK). (2017).

45. Karalunas, S. L., Geurts, H. M., Konrad, K., Bender, S. \& Nigg, J. T. Reaction time variability in ADHD and autism spectrum disorders: measurement and mechanisms of a proposed trans-diagnostic phenotype. J. Child Psychol. Psychiatry 55, 685-710 (2014).

46. American Psychiatric Association. Diagnostic and Statistical Manual, 4th edition (DSM-IV). (American Psychiatric Association, 1994).

47. Bussing, R. et al. Parent and teacher SNAP-IV ratings of Attention Deficit Hyperactivity Disorder symptoms: Psychometric properties and normative ratings from a school district sample. Assessment 15, 317-328 (2008).

48. Brainard, D. H. The Psychophysics Toolbox. Spat. Vis. 10, 433-436 (1997).

49. Pelli, D. G. The VideoToolbox software for visual psychophysics: transforming numbers into movies. Spat. Vis. 10, 437-442 (1997).

50. Evans, N. J., Steyvers, M. \& Brown, S. D. Modeling the covariance structure of complex datasets using cognitive models: An application to individual differences and the heritability of cognitive ability. Cogn. Sci. 42, 1925-1944 (2018).

51. Howard, Z. L., Evans, N. J., Innes, R. J., Brown, S. D. \& Eidels, A. How is multitasking different from increased difficulty? Psychon. Bull. Rev. 27, 937-951 (2020).

52. Ulrich, R. \& Miller, J. Effects of truncation on reaction time analysis. J. Exp. Psychol. Gen. 123, 34-80 (1994).

53. Evans, N. J. \& Brown, S. D. People adopt optimal policies in simple decision-making, after practice and guidance. Psychon. Bull. Rev. 24, 597-606 (2017). 
54. Evans, N. J. \& Hawkins, G. E. When humans behave like monkeys: Feedback delays and extensive practice increase the efficiency of speeded decisions. Cognition 184, 11-18 (2019).

55. Evans, N. J., Bennett, A. J. \& Brown, S. D. Optimal or not; depends on the task. Psychon. Bull. Rev. 26, 1027-1034 (2019).

56. Evans, N. J. Assessing the practical differences between model selection methods in inferences about choice response time tasks. Psychon. Bull. Rev. 26, 1070-1098 (2019).

57. Braak, C. J. F. T. A Markov Chain Monte Carlo version of the genetic algorithm Differential Evolution: easy Bayesian computing for real parameter spaces. Stat. Comput. 16, 239-249 (2006).

58. Turner, B. M., Sederberg, P. B., Brown, S. D. \& Steyvers, M. A method for efficiently sampling from distributions with correlated dimensions. Psychol. Methods 18, 368-384 (2013).

59. Morey, R. D. \& Rouder, J. N. BayesFactor: Computation of Bayes Factors for Common Designs. $R$ package version 0.9.12-4.2. (2018).

60. Delorme, A. \& Makeig, S. EEGLAB: an open source toolbox for analysis of singletrial EEG dynamics including independent component analysis. J. Neurosci. Methods 134, 9-21 (2004).

61. Manning, C. et al. Neural dynamics underlying coherent motion perception in children and adults. Dev. Cogn. Neurosci. 38, 100670 (2019).

62. Chang, C.-Y., Hsu, S.-H., Pion-Tonachini, L. \& Jung, T.-P. Evaluation of artifact subspace reconstruction for automatic EEG artifact removal. in 2018 40th Annual International Conference of the IEEE Engineering in Medicine and Biology Society (EMBC) 1242-1245 (2018). doi:10.1109/EMBC.2018.8512547. 
63. Dmochowski, J., Sajda, P., Dias, J. \& Parra, L. Correlated components of ongoing EEG point to emotionally laden attention - A possible marker of engagement? Front. Hum. Neurosci. 6, 112 (2012).

64. Dmochowski, J. P. \& Norcia, A. M. Cortical components of reaction-time during perceptual decisions in humans. PLOS ONE 10, e0143339 (2015).

65. O’Connell, R. G., Dockree, P. M. \& Kelly, S. P. A supramodal accumulation-tobound signal that determines perceptual decisions in humans. Nat. Neurosci. 15, 17291735 (2012).

66. Kelly, S. P. \& O’Connell, R. G. Internal and external influences on the rate of sensory evidence accumulation in the human brain. J. Neurosci. 33, 19434-19441 (2013).

67. Ehinger, B. V. \& Dimigen, O. Unfold: an integrated toolbox for overlap correction, non-linear modeling, and regression-based EEG analysis. PeerJ 7, e7838 (2019).

68. Kristensen, E., Guerin-Dugué, A. \& Rivet, B. Regularization and a general linear model for event-related potential estimation. Behav. Res. Methods 49, 2255-2274 (2017).

69. JASP Team. JASP (Version 0.14.1). (2020).

70. Turner, B. M., van Maanen, L. \& Forstmann, B. U. Informing cognitive abstractions through neuroimaging: the neural drift diffusion model. Psychol. Rev. 122, 312-336 (2015).

71. Turner, B. M. et al. A Bayesian framework for simultaneously modeling neural and behavioral data. NeuroImage 72, 193-206 (2013).

72. Turner, B. M., Rodriguez, C. A., Norcia, T. M., McClure, S. M. \& Steyvers, M. Why more is better: Simultaneous modeling of EEG, fMRI, and behavioral data. NeuroImage 128, 96-115 (2016).

73. Knowles, J. P., Evans, N. J. \& Burke, D. Some evidence for an association between early life adversity and decision urgency. Front. Psychol. 10, 243 (2019). 
74. Gelman, A. \& Rubin, D. B. Inference from iterative simulation using multiple sequences. Stat. Sci. 7, 457-472 (1992).

75. Jeffreys, H. The Theory of Probability, 3rd Edn. (Oxford University Press, 1961).

76. Lee, M. D. \& Wagenmakers, E.-J. Bayesian Cognitive Modeling: A Practical Course. (Cambridge University Press, 2014).

77. Del Viva, M. M., Igliozzi, R., Tancredi, R. \& Brizzolara, D. Spatial and motion integration in children with autism. Vision Res. 46, 1242-1252 (2006).

78. Jones, C. R. G. et al. No evidence for a fundamental visual motion processing deficit in adolescents with autism spectrum disorders. Autism Res. 4, 347-357 (2011).

79. Large, I., Pellicano, E., Mojzisch, A. \& Krug, K. Developmental trajectory of social influence integration into perceptual decisions in children. Proc. Natl. Acad. Sci. 116, 2713-2722 (2019).

80. Mulder, M. J. et al. Basic impairments in regulating the speed-accuracy tradeoff predict symptoms of Attention-Deficit/Hyperactivity Disorder. Biol. Psychiatry 68, 1114$1119(2010)$.

81. Boehm, U., Marsman, M., Matzke, D. \& Wagenmakers, E.-J. On the importance of avoiding shortcuts in applying cognitive models to hierarchical data. Behav. Res. Methods 50, 1614-1631 (2018).

82. Evans, N. J. \& Wagenmakers, E.-J. Theoretically meaningful models can answer clinically relevant questions. Brain 142, 1172-1175 (2019).

83. Tagliabue, C. F. et al. The EEG signature of sensory evidence accumulation during decision formation closely tracks subjective perceptual experience. Sci. Rep. 9, 4949 (2019).

84. Kiani, R. \& Shadlen, M. N. Representation of confidence associated with a decision by neurons in the parietal cortex. Science 324, 759-764 (2009). 
85. Gherman, S. \& Philiastides, M. G. Neural representations of confidence emerge from the process of decision formation during perceptual choices. NeuroImage 106, 134-143 (2015).

86. Williams, D. M., Bergström, Z. \& Grainger, C. Metacognitive monitoring and the hypercorrection effect in autism and the general population: Relation to autism(-like) traits and mindreading. Autism 22, 259-270 (2018).

87. Sahuquillo-Leal, R. et al. Jumping to conclusions in autism: integration of contextual information and confidence in decision-making processes. Eur. Child Adolesc. Psychiatry 29, 959-968 (2020).

88. Grainger, C., Williams, D. M. \& Lind, S. E. Metacognitive monitoring and control processes in children with autism spectrum disorder: Diminished judgement of confidence accuracy. Conscious. Cogn. 42, 65-74 (2016).

89. Cisek, P. Cortical mechanisms of action selection: the affordance competition hypothesis. Philos. Trans. R. Soc. B Biol. Sci. 362, 1585-1599 (2007).

90. Cisek, P. \& Kalaska, J. F. Neural mechanisms for interacting with a world full of action choices. Annu. Rev. Neurosci. 33, 269-298 (2010).

91. Metin, B. et al. ADHD performance reflects inefficient but not impulsive information processing: A diffusion model analysis. Neuropsychology 27, 193-200 (2013).

92. Weigard, A. \& Huang-Pollock, C. A diffusion modeling approach to understanding contextual cueing effects in children with ADHD. J. Child Psychol. Psychiatry 55, 1336$1344(2014)$.

93. Weigard, A. \& Huang-Pollock, C. The role of speed in ADHD-related working memory deficits: A time-based resource-sharing and diffusion model account. Clin. Psychol. Sci. 5, 195-211 (2017). 
94. Swanson, J. M. et al. Categorical and dimensional definitions and evaluations of symptoms of ADHD: History of the SNAP and the SWAN Rating Scales. Int. J. Educ. Psychol. Assess. 10, 51-70 (2012).

95. Pellicano, E. \& Gibson, L. Y. Investigating the functional integrity of the dorsal visual pathway in autism and dyslexia. Neuropsychologia 46, 2593-2596 (2008).

96. Benassi, M., Simonelli, L., Giovagnoli, S. \& Bolzani, R. Coherence motion perception in developmental dyslexia: a meta-analysis of behavioral studies. Dyslexia 16, 341-357 (2010).

\section{Acknowledgements}

We thank the participants and families who took part, the schools and organisations who kindly advertised the study, Irina Lepadatu, the Oxford Babylab and Dhea Bengardi for help with recruitment, and Helena Wood, Lisa Toffoli, Madeleine Mills, Amber Heaton, and Kate Seaborne who helped with data collection and data entry. The project was funded by a Sir Henry Wellcome Postdoctoral Fellowship awarded to CM (grant number 204685/Z/16/Z) and a James S. McDonnell Foundation Understanding Human Cognition Scholar Award to GS. NJE was supported by an Australian Research Council Discovery Early Career Researcher Award (DE200101130). We are grateful to Dorothy Bishop for providing funding for research assistance. A CC BY or equivalent license is applied to the Author Accepted Manuscript arising from this submission in accordance with the Wellcome Trust grant's open access conditions.

\section{Author Contributions}


CM, AMN, EJW \& GS conceptualised and designed the study. CM collected the data. CM and NJE analysed the data. CDH, LTH and AMN provided analysis tools. CM, GS and NJE drafted the manuscript and all authors reviewed the manuscript.

\section{Additional Information}

\section{Competing Interests}

The authors declare no competing interests. 


\section{Legends}

Figure 1. Schematic representation of the main diffusion model parameters

Within the diffusion model, the decision-making process is represented as a noisy evidence accumulation process originating from a starting point, $z$, towards one of two decision bounds (e.g., "left" and "right" directions in the motion tasks used in this study). Boundary separation, $a$, reflects the distance between the two bounds, indexing response caution. Driftrate, $v$, is the rate of evidence accumulation. Non-decision time, ter, is the time taken for nondecision processes including sensory encoding and response generation.

Table 1. Demographics of participants included in final dataset

\section{Figure 2. Schematic representation of trial procedure.}

An initial fixation period was followed by a random motion period consisting of randomly moving dots. Next, the stimulus period contained leftward or rightward global motion and lasted until the child reported the direction with a response box. If there was no response, the stimulus remained on the screen for $2500 \mathrm{~ms}$. The stimulus remained on the screen for an offset period after the response or after the maximum stimulus duration was reached. Note that arrows (indicating movement) and dotted lines (marking the square stimulus region) are shown for illustrative purposes only. Figure reproduced from https://osf.io/wmtpx/ under a CC-BY4.0 license.

Figure 3. Response-locked evoked potentials for the motion coherence task and direction integration task 
Topographic maps represent the forward-model projections of the most reliable component reflecting the weights given to each electrode following reliable components analysis (RCA) on data from all participants pooled across difficulty level for the motion coherence task (top) and direction integration task (bottom). Each individual's continuous data were multiplied by these weights to provide a component average waveform, with group average responselocked waveforms $( \pm 1 \mathrm{SEM})$ shown for typically developing children (TD; grey) and autistic children (orange) for difficult and easy levels. The left column shows non-deconvolved group average waveforms. The central column shows deconvolved group average waveforms (without regularisation). The right column shows deconvolved group average waveforms with regularisation (ridge regression).

\section{Figure 4. Accuracy and median response time (RT) for correct trials}

Violin plots showing the kernel probability density for each group's accuracy (left) and median RT (in seconds) for correct trials (right) for each difficulty level and each task (upper: motion coherence; lower: direction integration). Data for typically developing children and autistic children are presented in grey and orange, respectively. Dots and vertical lines represent the group mean and \pm 1 SEM.

\section{Figure 5. Prior and posterior density distributions}

Prior (blue) and posterior (purple) density distributions for the group-level parameters reflecting group differences in each of the 5 model parameters $(\mathrm{v} \cdot \mathrm{mean}=$ mean drift rate across difficulty levels; $\mathrm{a}=$ boundary separation; ter $=$ non-decision time; v.diff $=$ difference in mean drift rate between difficulty levels; $\mathrm{z} / \mathrm{a}=$ relative starting point) for each task. Inset is a schematic representation of the model parameters. Columns 1 and 2 show the results of the 
standard model and Columns 3 and 4 show the results of the model with performance IQ partialled out. Positive values reflect higher parameter values in the autistic group compared to the typically developing group. $\mathrm{BF}=$ Savage-Dickey Bayes factors in favour of the alternative hypothesis over the null hypothesis, where BF > 1 reflect more support for the alternative hypothesis.

\section{Figure 6. EEG slope measure extracted for inclusion in the joint model}

Violin plots showing the kernel probability density for the EEG slope measure extracted for inclusion in the joint model for each group (typically developing: grey; autistic: orange) for each difficulty level. The extracted measure was the slope of a linear regression line fitted to each participant's deconvolved (with regularisation) response-locked waveform, from $200 \mathrm{~ms}$ prior to the response to the response (see shaded area of schematic response-locked waveform in inset). The dotted line reflects a flat slope. Dots and vertical lines represent the group mean and \pm 1 SEM.

Figure 7. Posterior density plots showing the correlation between drift-rate and the

\section{EEG measure}

Inset provides a schematic representation of the drift-rate parameter $(v ;$ left $)$ and EEG measure (slope of response-locked waveform from -200 ms to $0 \mathrm{~ms}$ around the response; right) that were correlated in the joint model, where $\rho$ represents the correlation. Posterior density plots in the left column reflect the correlation between the mean drift-rate across difficulty levels (v.mean) and the mean EEG slope measure across difficulty levels (EEG.mean). Posterior density plots in the right column reflect the correlation between the difference in drift-rate between difficulty levels (v.diff) and the difference in EEG slope 
measure between difficulty levels (EEG.diff). Plots for the motion coherence task are presented in the upper row and plots for the direction integration task are presented in the lower row. The blue distribution shows the correlation across all participants, and the grey and orange distributions show separate correlations estimated for typically developing children and autistic children, respectively. 


\section{Supplementary Materials relating to Manning et al. 'Behavioural and neural indices of perceptual decision-making in autistic children during visual motion tasks'}
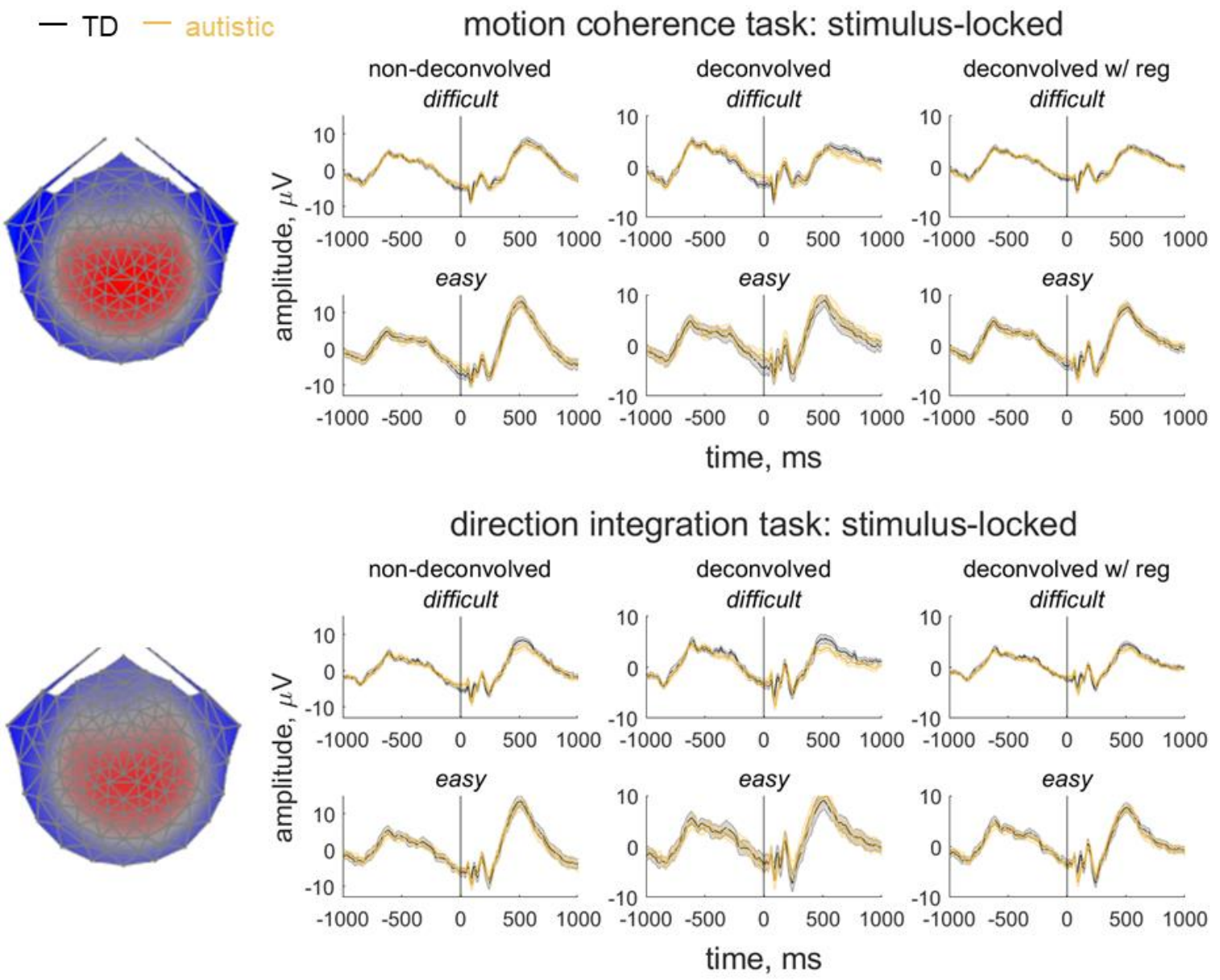

Figure S1. Stimulus-locked evoked potentials for the motion coherence task and direction integration task

Topographic maps represent the forward-model projections of the most reliable component reflecting the weights given to each electrode following reliable components analysis (RCA) on data from all participants pooled across difficulty level for the motion coherence task (top) and direction integration task (bottom). Each individual's continuous data were multiplied by these weights to provide a component average waveform, with group average stimulus-locked waveforms ( $\pm 1 \mathrm{SEM}$ ) shown for typically developing children (TD; grey) and autistic children (orange) for difficult and easy levels. The left column shows non-deconvolved group average waveforms. The central column shows deconvolved group average waveforms (without regularisation). The right column shows deconvolved group average waveforms with regularisation (ridge regression). 


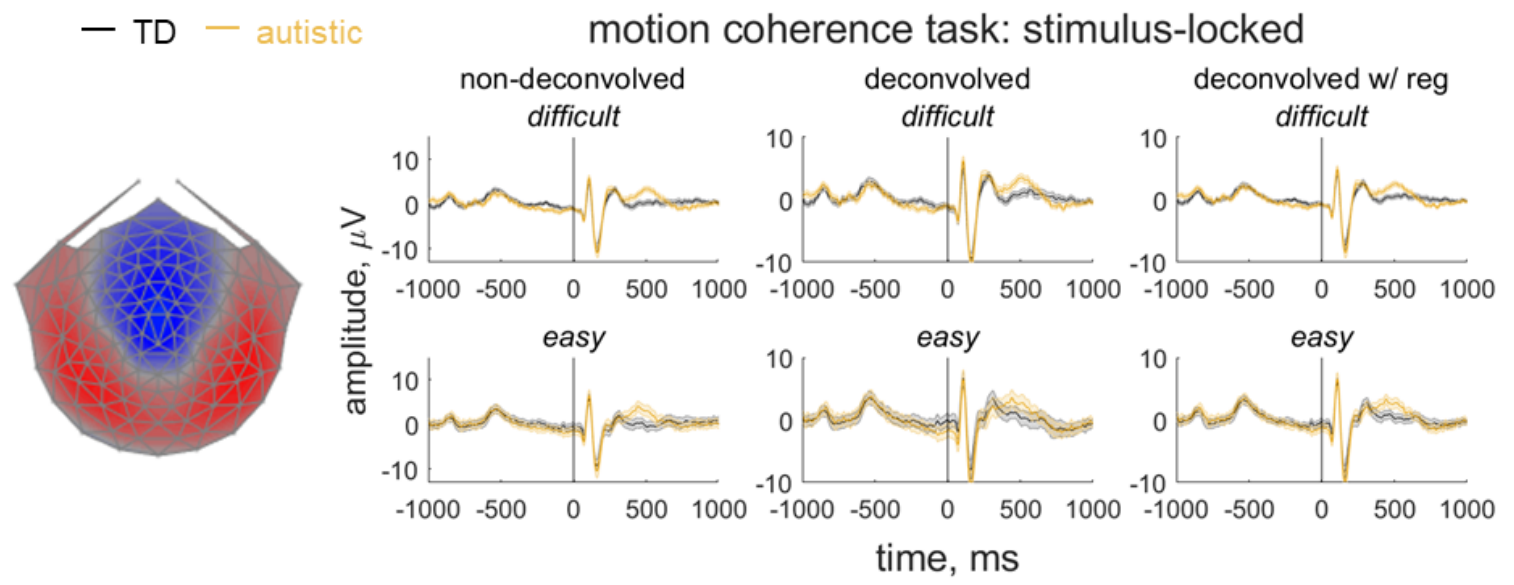

direction integration task: stimulus-locked
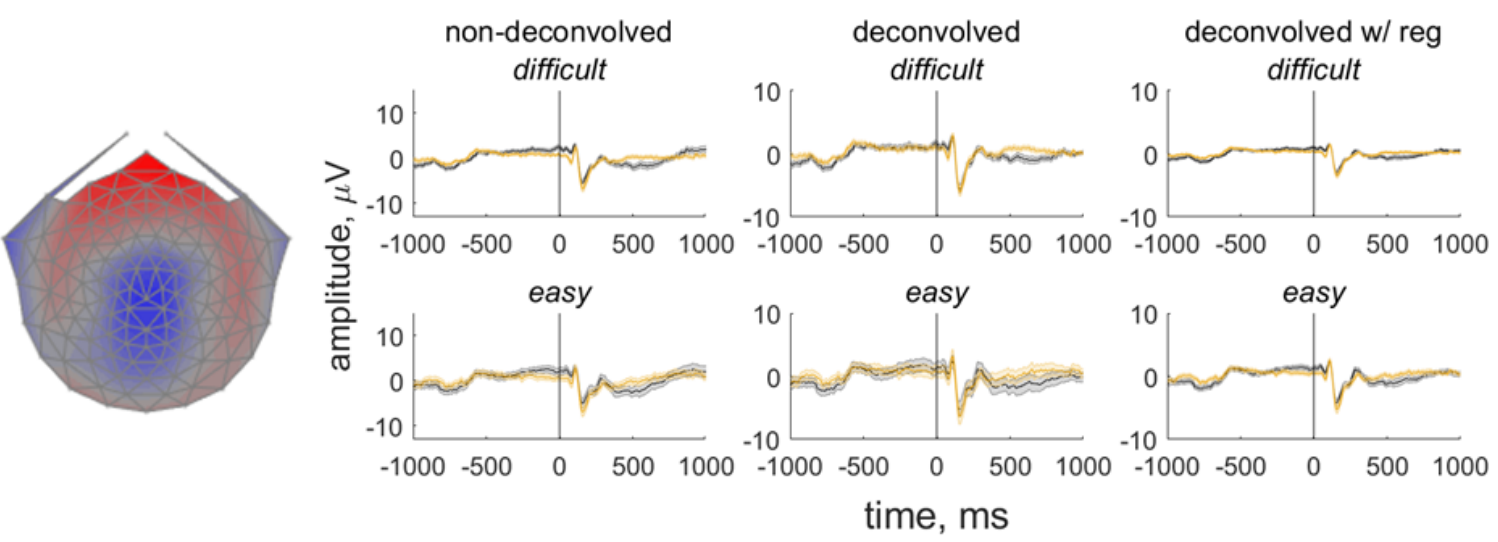

Figure S2. Stimulus-locked evoked potentials for the motion coherence task and direction integration task for the second-most reliable component (RC2)

Topographic maps represent the forward-model projections of the second-most reliable component reflecting the weights given to each electrode following reliable components analysis (RCA) on data from all participants pooled across difficulty level for the motion coherence task (top) and direction integration task (bottom). Each individual's continuous data were multiplied by these weights to provide a component average waveform, with group average stimulus-locked waveforms $( \pm 1$ SEM) shown for typically developing children (TD; grey) and autistic children (orange) for difficult and easy levels. The left column shows non-deconvolved group average waveforms. The central column shows deconvolved group average waveforms (without regularisation). The right column shows deconvolved group average waveforms with regularisation (ridge regression). The autistic children and typically developing children show remarkably overlapping waveforms in early stages of processing (over the first $\sim 400 \mathrm{~ms}$ following stimulus onset, see also [35]). Note that the topographic maps and waveform peaks are less defined in the direction integration task than in the motion coherence task. 


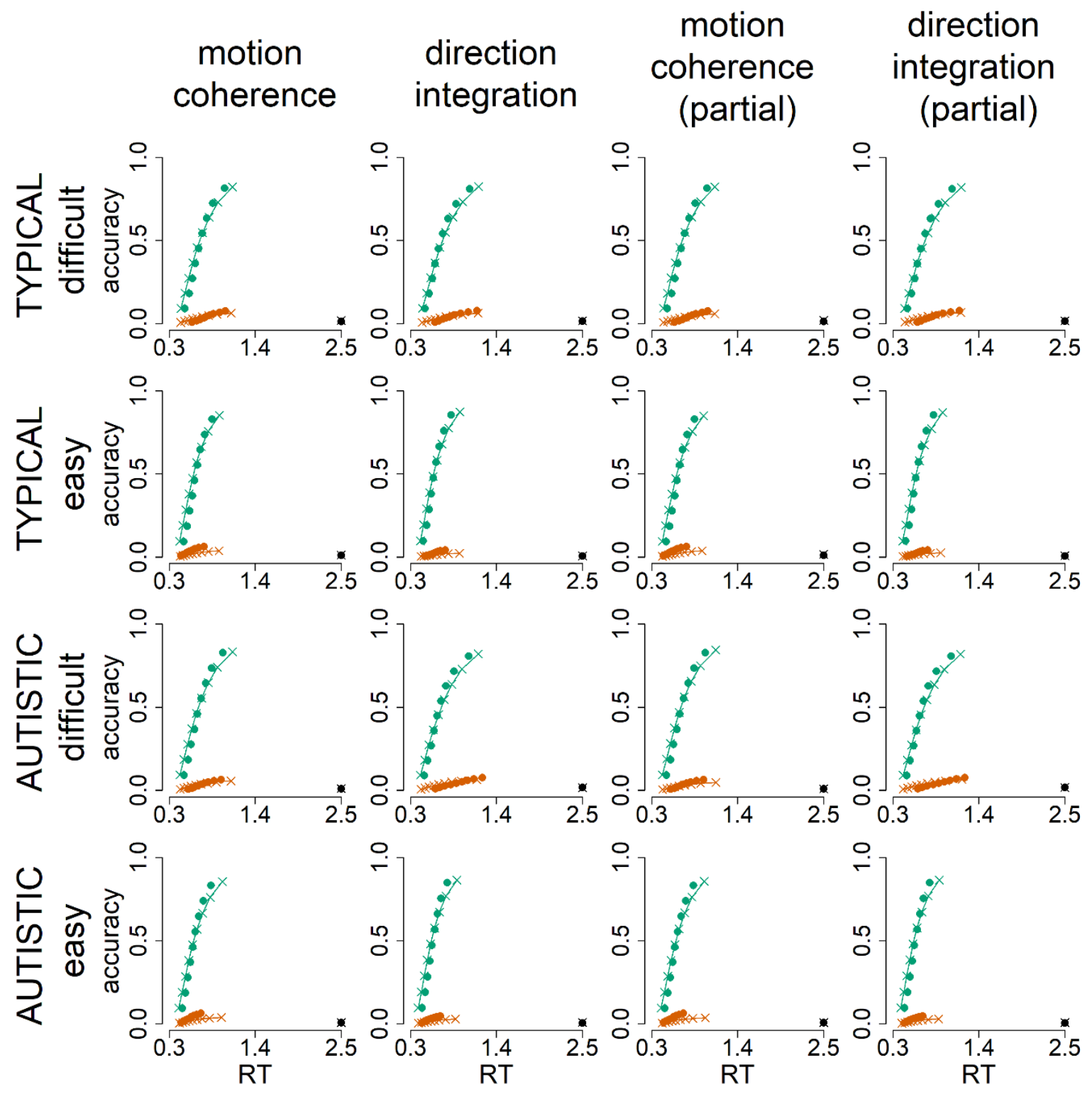

\section{Figure S3. Model fits}

Defective cumulative density function plots for each of the four models, for typically developing children (upper rows) and autistic children (bottom rows) for difficult and easy levels. Green represents correct responses and red represents error responses, at each of 9 quantiles. The dots reflect the observed data and crosses with connecting lines reflect the model fit. The dots and crosses at 2.5 seconds reflect the observed and model predicted misses. 


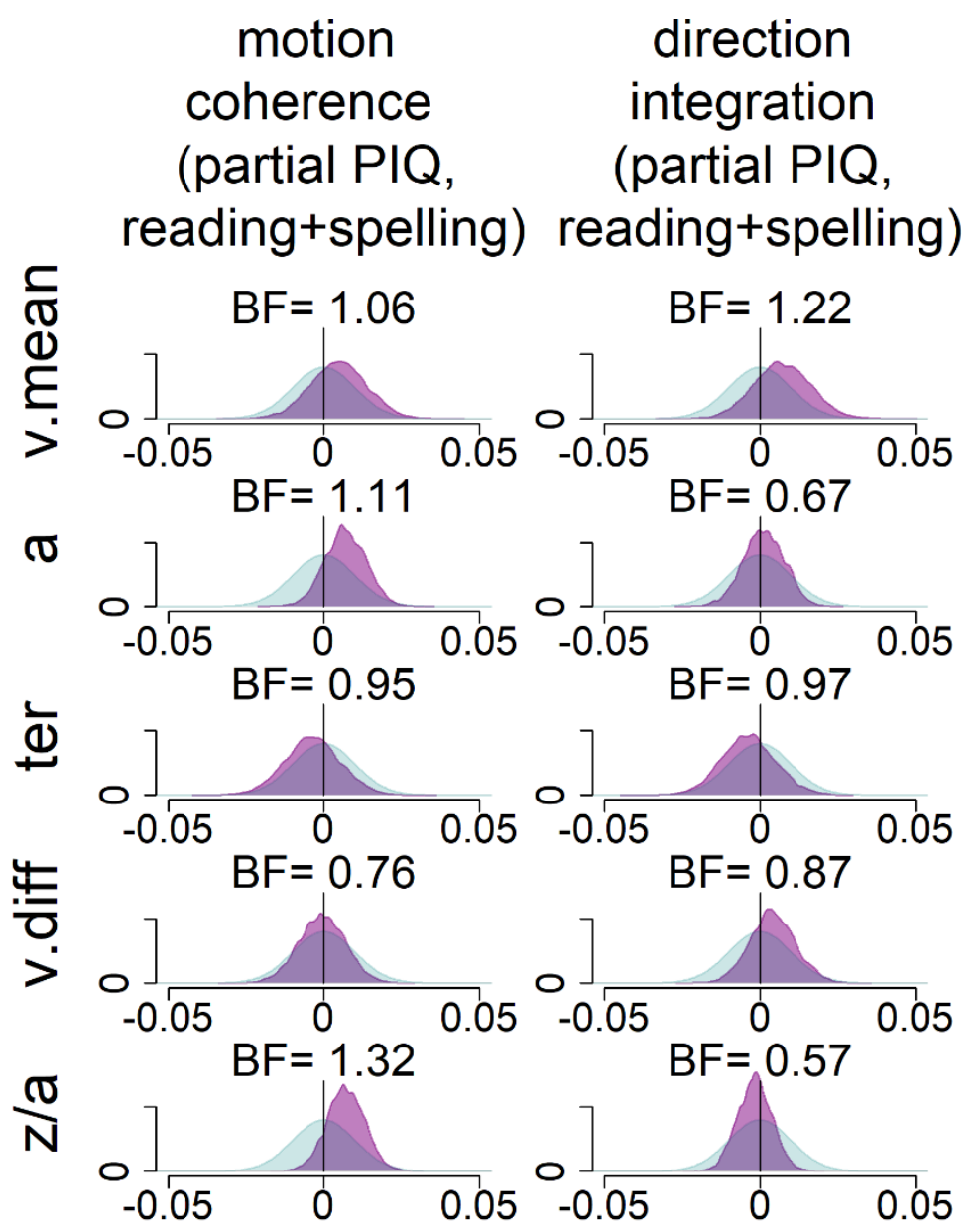

Figure S4. Prior and posterior density distributions for model with reading ability, spelling ability and performance IQ partialled out 


\section{motion coherence}
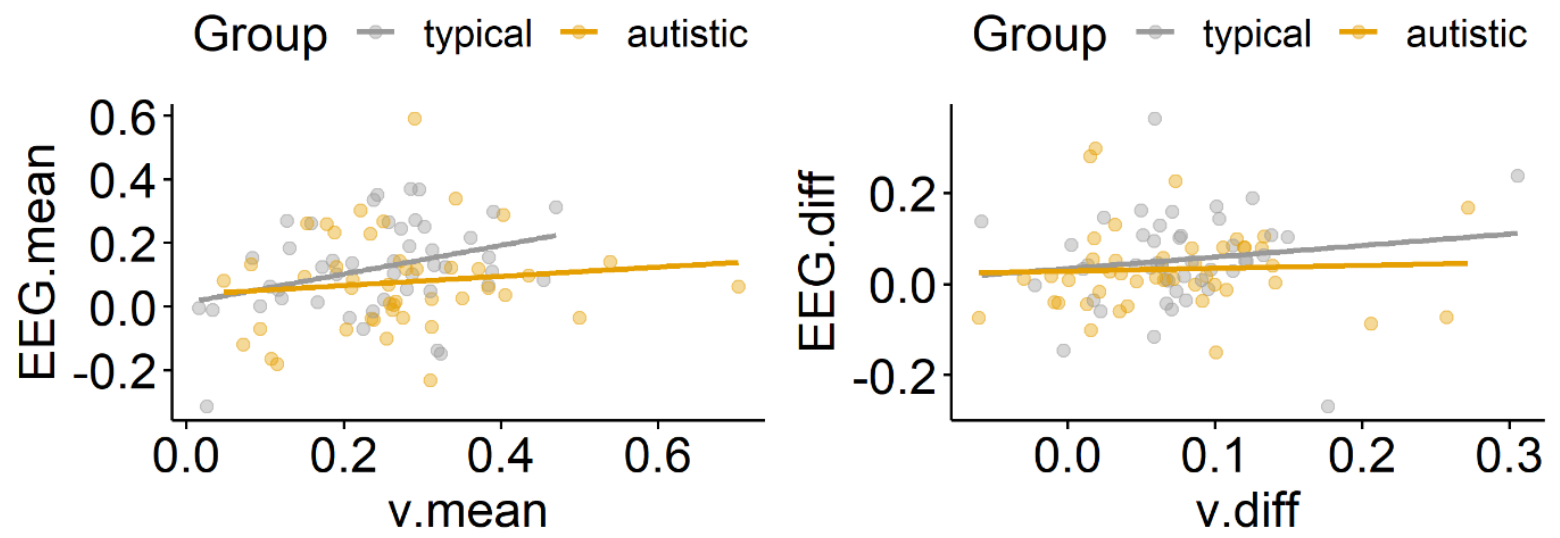

\section{direction integration}
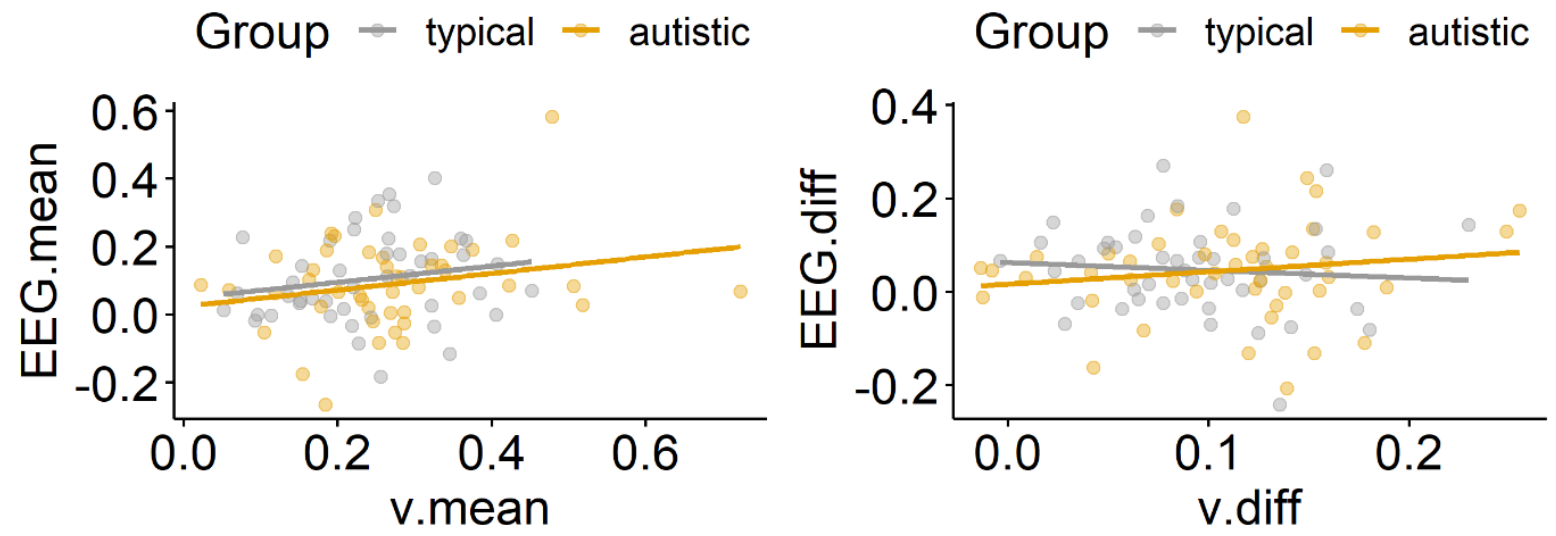

Figure S5. Scatterplots showing relationship between drift-rate and EEG

Left panels show maximum likelihood estimates contained within the posterior for each participant's mean drift-rate across difficulty levels (v.mean) plotted against the slope of EEG activity averaged across difficulty levels (EEG.mean) for the motion coherence (top) and direction integration (bottom) tasks. Right panels show point estimates for each participant's difference in drift-rate between difficulty levels ( $v$.diff) plotted against the difference in slopes of EEG activity between the two difficulty levels (EEG.diff), for each task. Typically developing children are plotted in grey and children with dyslexia are plotted in blue. 

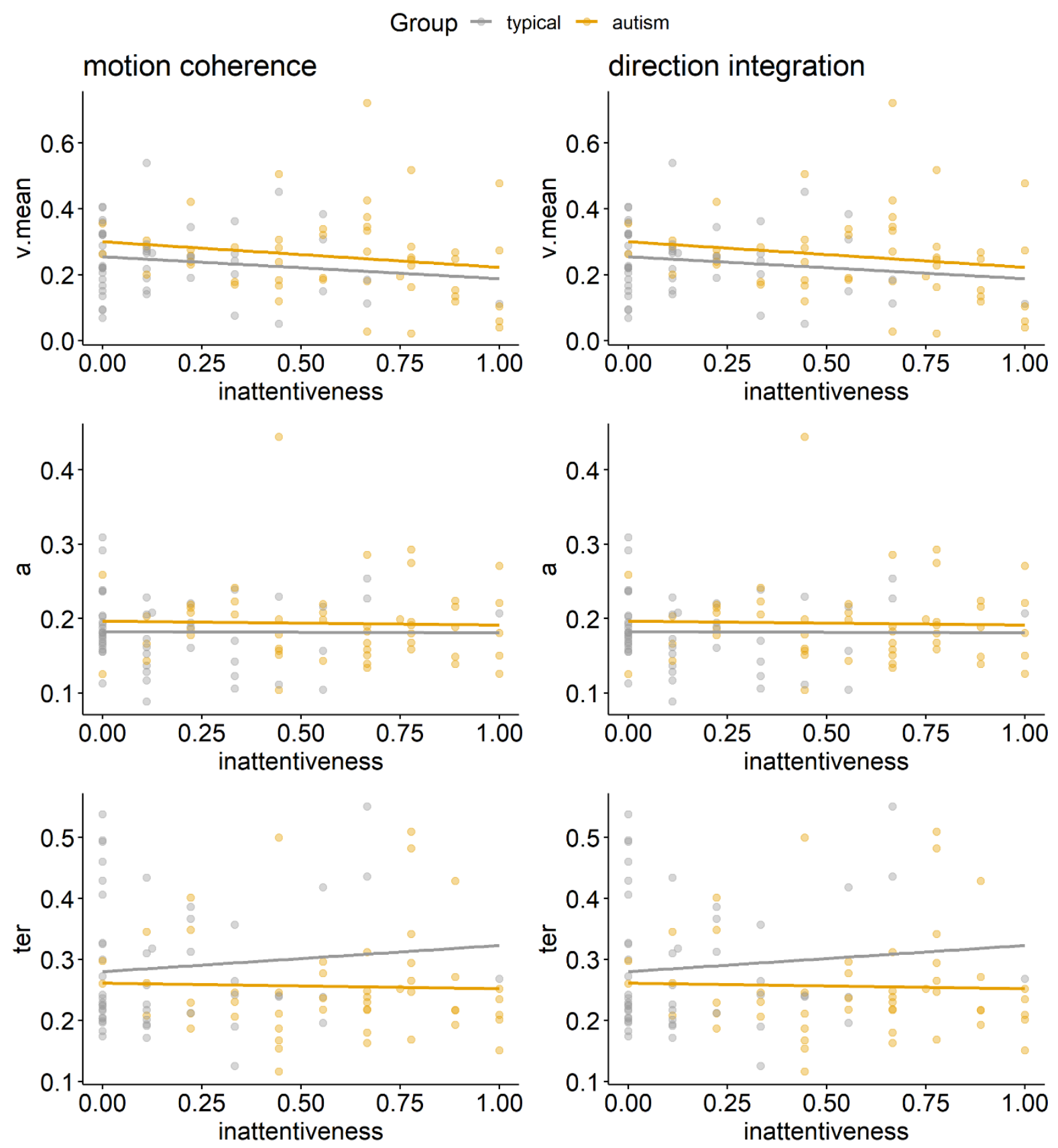

Figure S6. Scatterplots showing relationships between main diffusion model parameters and inattentiveness scores 

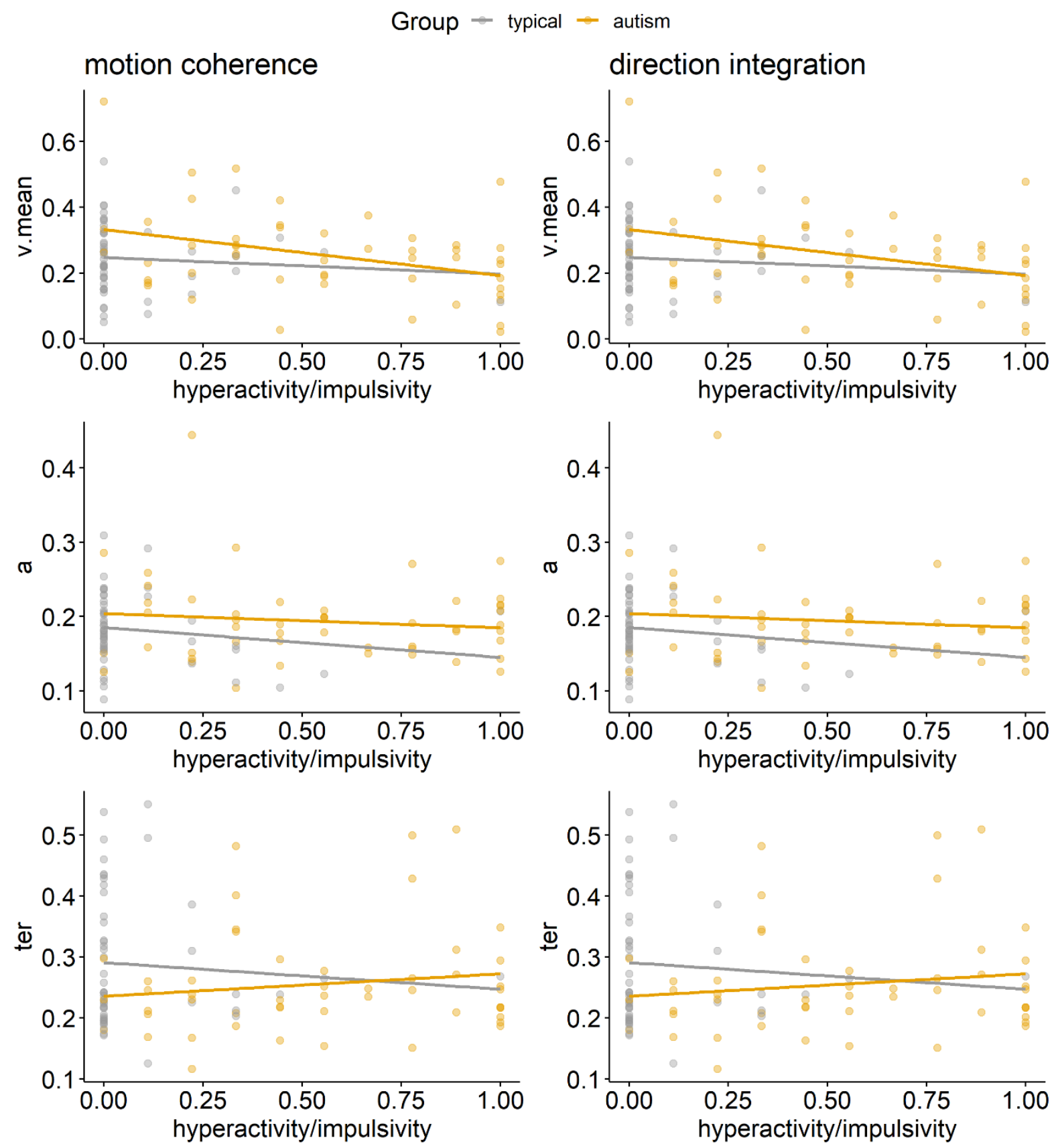

Figure S7. Scatterplots showing relationships between main diffusion model parameters and hyperactivity/impulsivity scores 
motion coherence v.mean \& inattentiveness

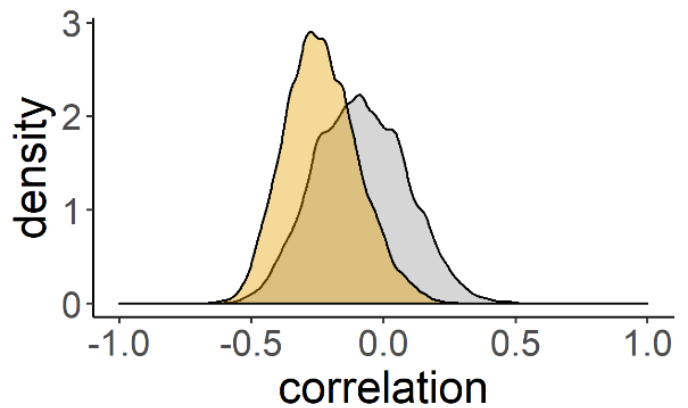

direction integration v.mean \& inattentiveness

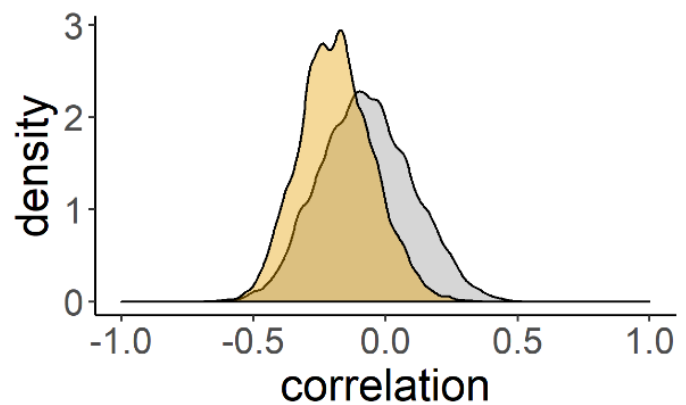

motion coherence v.mean \& hyperactivity

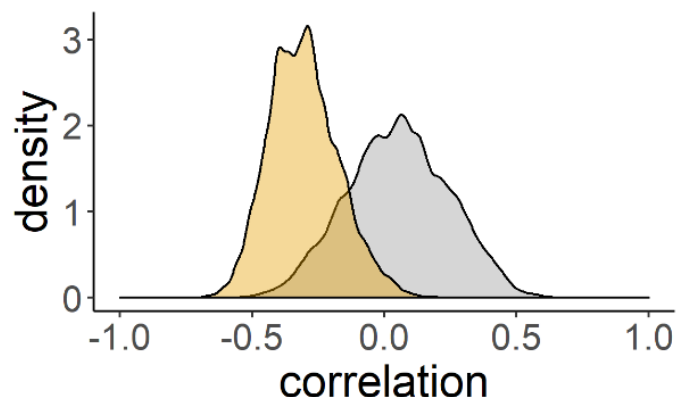

Group

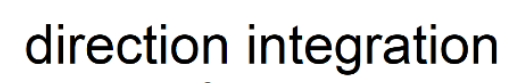
v.mean \& hyperactivity

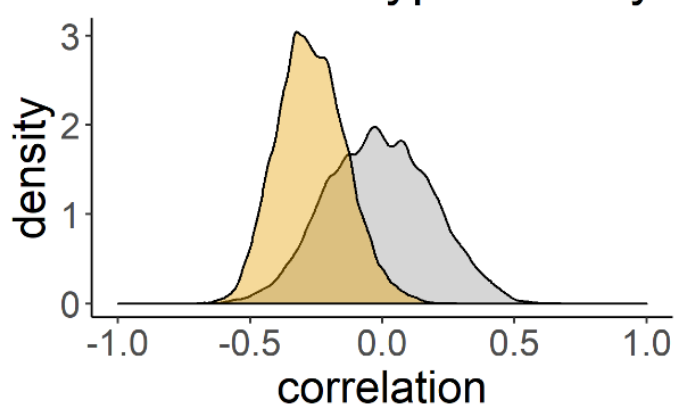

Figure S8. Posterior density plots showing correlations between mean drift-rate across difficulty levels (v.mean) and parent-reported ADHD symptoms

Plots for the motion coherence task are presented in the upper row and plots for the direction integration task are presented in the lower row. The left column shows the relationship between mean drift-rate (v.mean) and inattentiveness, and the right column shows the relationship between mean drift-rate (v.mean) and hyperactivity/impulsivity. The grey and orange distributions show separate correlations estimated for typically developing and autistic children, respectively. 
motion coherence a \& inattentiveness

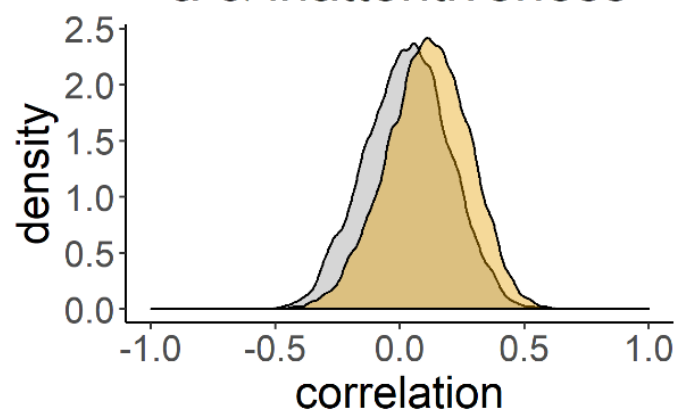

direction integration a \& inattentiveness

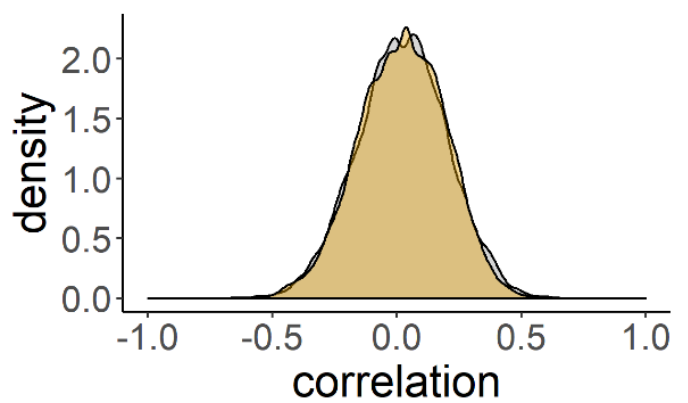

motion coherence

a \& hyperactivity

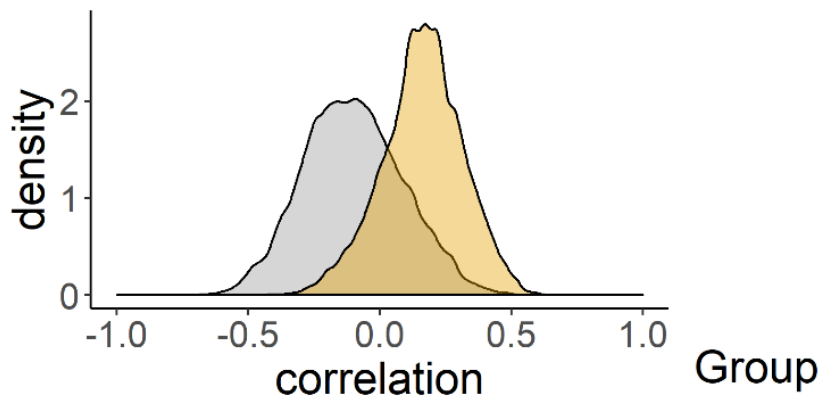

direction integration a \& hyperactivity

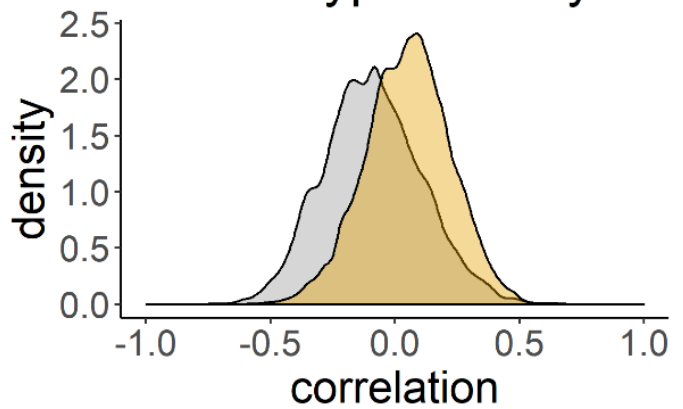

Figure S9. Posterior density plots showing correlations between boundary separation (a) and parent-reported ADHD symptoms

Plots for the motion coherence task are presented in the upper row and plots for the direction integration task are presented in the lower row. The left column shows the relationship between boundary separation (a) and inattentiveness, and the right column shows the relationship between boundary separation (a) and hyperactivity/impulsivity. The grey and orange distributions show separate correlations estimated for typically developing and autistic children, respectively. 
motion coherence ter \& inattentiveness

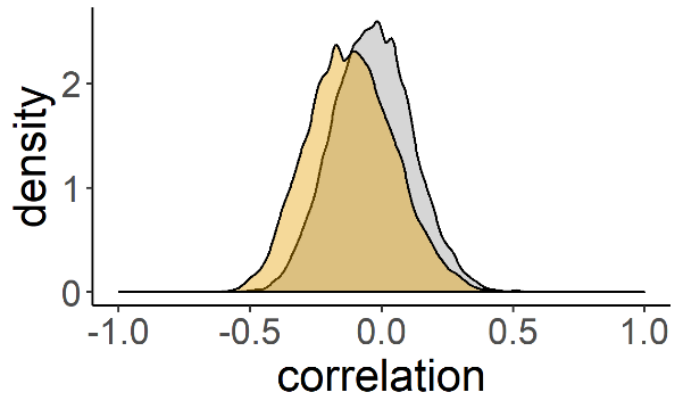

direction integration ter \& inattentiveness

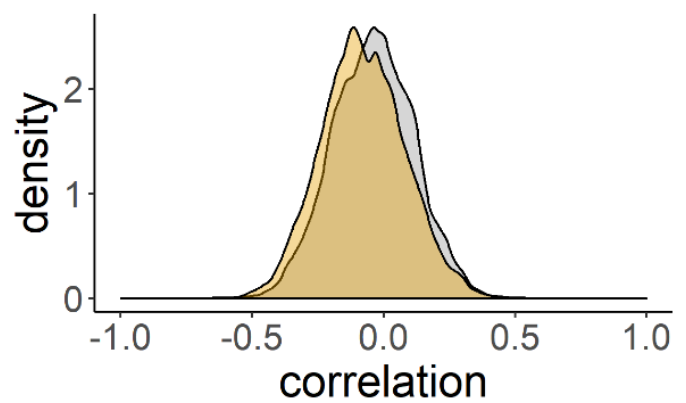

motion coherence

ter \& hyperactivity

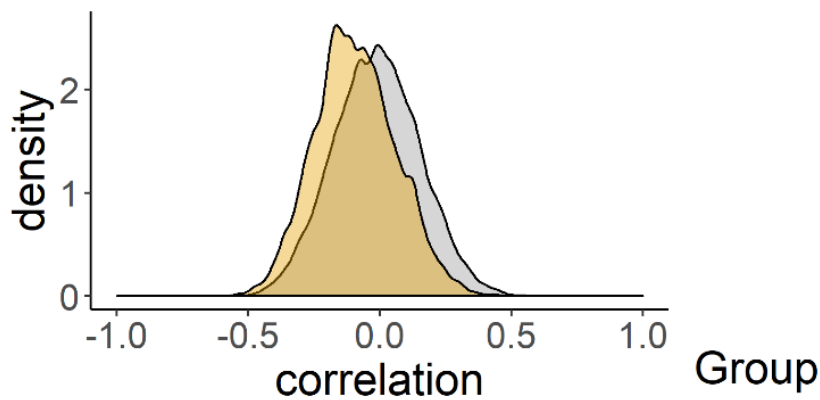

direction integration ter \& hyperactivity

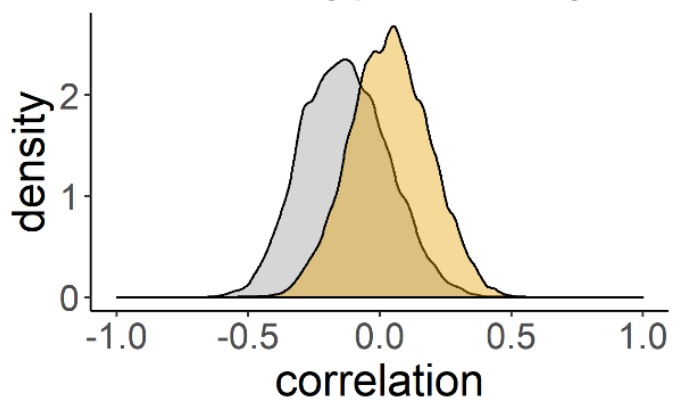

Figure S10. Posterior density plots showing correlations between non-decision time (ter) and parent-reported ADHD symptoms

Plots for the motion coherence task are presented in the upper row and plots for the direction integration task are presented in the lower row. The left column shows the relationship between non-decision time (ter) and inattentiveness, and the right column shows the relationship between non-decision time (ter) and hyperactivity/impulsivity. The grey and orange distributions show separate correlations estimated for typically developing and autistic children, respectively. 
Table S1. Statistics summarising posterior distributions for the correlations between parent-reported ADHD symptoms (hyperactivity/impulsivity and inattentiveness) and diffusion model parameters, for each group, in each task.

\begin{tabular}{|c|c|c|c|c|}
\hline \multirow[t]{2}{*}{ Corr } & \multicolumn{2}{|c|}{ Motion coherence task } & \multicolumn{2}{|c|}{ Direction integration task } \\
\hline & Typical & Autistic & Typical & Autistic \\
\hline \multicolumn{5}{|c|}{ Hyperactivity/impulsivity, } \\
\hline v.mean & $.05[-.32, .42], .27$ & $-.31[-.54,-.02], 1.82$ & $-.01[-.39, .37], .27$ & $-.27[-.51,0], 1.27$ \\
\hline $\mathrm{a}$ & $-.10[-.46, .28], .30$ & $.16[-.16, .45], .38$ & $-.09[-.45, .31], .29$ & $.05[-.29, .37], .24$ \\
\hline ter & $-.01[-.33, .31], .21$ & $-.09[-.38, .22], .25$ & $-.13[-.43, .19], .30$ & $.04[-.26, .33], .21$ \\
\hline \multicolumn{5}{|c|}{ Inattentiveness, } \\
\hline v.mean & $-.09[-.41, .25], .27$ & $-.23[-.48, .06], .68$ & $-.07[-.40, .27], .25$ & $-.19[-.45, .10], .47$ \\
\hline $\mathrm{a}$ & $.03[-.30, .35], .22$ & $.11[-.22, .42], .29$ & $.02[-.33, .37], .23$ & $.02[-.33, .35], .24$ \\
\hline ter & $-.04[-.32, .27], .20$ & $-.12[-.42, .22], .28$ & $-.04[-.34, .26], .20$ & $-.08[-.38, .24], .23$ \\
\hline
\end{tabular}

Note. Data are presented as posterior mean [95\% credible intervals], Bayes factor (BF) 